\title{
Multiply Warped Product Generalized Semi-Invariant Submanifolds
}

\author{
Moctar Traore, Hakan Mete Taştan and Sibel Gerdan Aydın* \\ (Communicated by Bang-Yen Chen)
}

\begin{abstract}
We define generalized semi-invariant submanifolds in locally product Riemannian manifolds. Then we study multiply warped product generalized semi-invariant submanifolds in the same structure. We give an existence theorem for such submanifolds. We also give necessary and sufficient conditions for such a submanifold to be a multiply direct product submanifold. Moreover, we establish a general inequality for such submanifolds.
\end{abstract}

Keywords: Multiply warped product submanifold, slant distribution, invariant distribution, anti-invariant distribution, generalized semi-invariant submanifold, locally product Riemannian manifold.

AMS Subject Classification (2020): Primary: 53C15 ; Secondary: 53B20

\section{Introduction}

Multiply warped product manifolds [12] are natural generalization of the warped product manifolds [8]. These notions play very important roles in physics as well as in differential geometry, especially in the theory of relativity. Indeed, the standard spacetimes models such as Roberston-Walker, Schwarschild, static and Kruskal are warped products. Also, the simplest models of neighborhoods of stars and black holes are warped product [16].

On the other hand, warped or multiply warped product submanifolds have been studying very actively since Chen [9] studied the warped product CR-submanifolds in Kaehler structures. The most of the studies related to the warped or multiply warped product submanifolds can be found in the book [11] and its list of references.

In this paper, motivated by the papers placed in [11], especially Chen and Dillen's paper [10], we study a certain type of multiply warped product submanifolds in locally product Riemannian manifolds. In particular, we consider the multiply warped product submanifolds in the form $M^{\theta} \times_{f_{1}} M_{1}^{T} \times \ldots \times_{f_{k}} M_{k}^{T} \times_{\sigma_{1}} M_{1}^{\perp} \times$ $\ldots \times_{\sigma_{l}} M_{l}^{\perp}$, where $M^{\theta}$ is a proper slant, $M_{i}^{T}$ is an invariant submanifold and $M_{j}^{\perp}$ is an anti-invariant submanifold of the locally product Riemannian manifold for $1 \leq i \leq k$ and $1 \leq j \leq l$. We give necessary and sufficient conditions for a generalized semi-invariant submanifold to be a locally multiply warped product in the main theorem. Also, we investigate the behavior of the second fundamental form of such submanifolds and as results, we give necessary and sufficient conditions for such submanifolds to be locally multiply direct or usual product and get an inequality for the squared norm of the second fundamental form in terms of the warping functions for such submanifolds. 


\section{Preliminaries}

In this section, we give the fundamental definitions and notions needed for further study. In subsection 2.1, we will recall the definition of the multiply warped product manifolds. In subsection 2.2 , we give the basic background for submanifolds of Riemannian manifolds. The definition of a locally product Riemannian manifold is placed in the last subsection.

\subsection{Multiply warped product manifolds}

Let $\left(M_{0}, g_{0}\right),\left(M_{1}, g_{1}\right), \ldots,\left(M_{k}, g_{k}\right)$ be Riemannian manifolds and let $f_{1}, f_{2}, \ldots, f_{k}$ be positive smooth functions on $M_{0}$. Then the multiply warped product manifold [12] $M_{0} \times_{f_{1}} M_{1} \times \ldots \times f_{k} M_{k}$ is the multiply product manifold $M_{0} \times M_{1} \times \ldots \times M_{k}$ furnished with the metric

$$
g=\pi_{0}^{*}\left(g_{0}\right) \oplus\left(f_{1} \circ \pi_{0}\right)^{2} \pi_{1}^{*}\left(g_{1}\right) \oplus \ldots \oplus\left(f_{k} \circ \pi_{0}\right)^{2} \pi_{k}^{*}\left(g_{k}\right) .
$$

More precisely, for any vector fields $\bar{X}$ and $\bar{Y}$ on $\bar{M}$, we have

$$
g(\bar{X}, \bar{Y})=g_{0}\left(\pi_{0_{*}} \bar{X}, \pi_{0_{*}} \bar{Y}\right)+\sum_{i=1}^{k}\left(f_{i} \circ \pi_{0}\right)^{2} g_{i}\left(\pi_{i_{*}} \bar{X}, \pi_{i_{*}} \bar{Y}\right),
$$

where $\pi_{i}: \bar{M}=M_{0} \times M_{1} \times \ldots \times M_{k} \rightarrow M_{i}, i=0,1, \ldots, k$ is the canonical projection, $\pi_{i}^{*}\left(g_{i}\right)$ is the pullback of $g_{i}$ via $\pi_{i}$ and the subscript ${ }^{*}$ denotes the derivative map of $\pi_{i}$. The functions $f_{1}, \ldots, f_{k}$ are called the warping functions of $M_{0} \times f_{1} M_{1} \times \ldots \times f_{k} M_{k}$. The manifolds $\left(M_{1}, g_{1}\right), \ldots,\left(M_{k}, g_{k}\right)$ are called the fibers and the manifold $\left(M_{0}, g_{0}\right)$ is called the base manifold of the multiply warped product manifold $M_{0} \times_{f_{1}} M_{1} \times \ldots \times{ }_{f_{k}} M_{k}$. It is well known that the base manifold is totally geodesic and the fibers are totally umbilic in $M_{0} \times f_{1} M_{1} \times \ldots \times f_{k} M_{k}$.

As mentioned in the previous section, the notion of the multiply warped product is a generalization of direct product as well as warped product manifolds. Indeed, if we choose $k=1$ in the definition above, then we get a warped product [8] and if each warping function $f_{i}$ is constant in the definition above, then we get a multiply direct product [11].

Let $M_{0} \times_{f_{1}} M_{1} \times \ldots \times_{f_{k}} M_{k}$ be a multiply warped product manifold with the Levi-Civita connection $\bar{\nabla}$ with respect to the metric $g$ given in (2.1) and $\nabla^{i}$ denote the Levi-Civita connection of $\left(M_{i}, g_{i}\right)$ for $i \in\{0,1, \ldots, k\}$. By usual convenience, we denote the set of lifts of vector fields on $M_{i}$ by $\mathcal{L}\left(M_{i}\right)$ and use the same notation for a vector field (resp. warping function) and its lift (resp. its pulback). On the other hand, since the map $\pi_{0}$ is an isometry and $\pi_{1}, \ldots, \pi_{k}$ are positive homotheties, they preserve the Levi-Civita connections. Thus there is no confusion using the same symbol for a connection on $M_{i}$ and for its pullback via $\pi_{i}$. Then, the covariant derivative formulas [23] of the multiply warped product manifold $M_{0} \times_{f_{1}} M_{1} \times \ldots \times_{f_{k}} M_{k}$ are given by

$$
\begin{gathered}
\bar{\nabla}_{Z} W=\nabla_{Z}^{0} W \\
\bar{\nabla}_{Z} X=\bar{\nabla}_{X} Z=Z\left(\ln f_{i}\right) X \\
\bar{\nabla}_{X} Y=\left\{\begin{array}{lll}
0 & \text { if } & i \neq j, \\
\nabla_{X}^{i} Y-g(X, Y) \nabla^{0}\left(\ln f_{i}\right) & \text { if } & i=j,
\end{array}\right.
\end{gathered}
$$

where $Z, W \in \mathcal{L}\left(M_{0}\right), X \in \mathcal{L}\left(M_{i}\right)$ and $Y \in \mathcal{L}\left(M_{j}\right)$ for $i, j \in\{1,2, \ldots, k\}$.

\subsection{Submanifolds of Riemannian manifolds}

Let $M$ be a Riemannian manifold isometrically immersed in a Riemannian manifold $(\bar{M}, g)$ and $\bar{\nabla}$ be the Levi-Civita connection of $\bar{M}$ with respect to the metric g. Also, let $\nabla$ and $\nabla^{\perp}$ be the induced and induced normal connection on $M$, respectively. Then the Gauss and Weingarten formulas [25] are given respectively by

$$
\bar{\nabla}_{V} W=\nabla_{V} W+h(V, W) \quad \text { and } \quad \bar{\nabla}_{V} Z=-A_{Z} V+\nabla_{V}^{\perp} Z
$$


where the vector fields $V, W$ are tangent to $M$ and $Z$ is normal to $M$. In addition, $h$ is the second fundamental form of $M$ and $A_{Z}$ is the Weingarten endomorphism associated with $Z$. The second fundamental form $h$ and the shape operator $A$ are related by

$$
g(h(V, W), Z)=g\left(A_{Z} V, W\right) .
$$

The mean curvature vector $H$ for an orthonormal frame $\left\{e_{1}, \ldots, e_{m}\right\}$ of tangent space $T_{p} M, p \in M$ on $M$ is defined by

$$
H=\frac{1}{m} \operatorname{trace}(h)=\frac{1}{m} \sum_{i=1}^{m} h\left(e_{i}, e_{i}\right)
$$

where $m=\operatorname{dim}(M)$. Also, we set

$$
h_{i j}^{r}=g\left(h\left(e_{i}, e_{j}\right), e_{r}\right) \quad \text { and } \quad\|h\|^{2}=\sum_{i, j=1}^{m} g\left(h\left(e_{i}, e_{j}\right), h\left(e_{i}, e_{j}\right)\right)
$$

$r=n-m$, where $n=\operatorname{dim}(\bar{M})$ and $m=\operatorname{dim}(M)$.

\subsection{Locally product Riemannian manifolds}

Let $\bar{M}$ be any manifold equipped with a tensor field of type $(1,1)$ such that

$$
F^{2}=I, \quad(F \neq \mp I)
$$

where $I$ is the identity endomorphism on the tangent bundle $T \bar{M}$ of $\bar{M}$. Then we say that $(\bar{M}, F)$ is an almost product manifold with almost product structure $F$. If the almost product manifold $(\bar{M}, F)$ admits a metric tensor $g$ such that

$$
g(F \bar{X}, F \bar{Y})=g(\bar{X}, \bar{Y})
$$

for all $\bar{X}, \bar{Y} \in \Gamma(T \bar{M})$, then $(\bar{M}, F, g)$ is called an almost product Riemannian manifold. Let $\bar{\nabla}$ be the Levi-Civita connection of $(\bar{M}, F, g)$, then we say that $(\bar{M}, F, g)$ is a locally product Riemannian manifold (briefly, l.p.R. manifold) or locally decomposable Riemannian manifold if $F$ is parallel with respect to $\bar{\nabla}$, i.e.

$$
\bar{\nabla}_{\bar{X}} F \equiv 0
$$

for all $\bar{X} \in \Gamma(T \bar{M})[25]$.

\section{Generalized semi-invariant submanifolds in locally product Riemannian manifolds}

In this section, we define the definition of the generalized semi-invariant submanifolds of a 1.p.R. manifold and get some useful results for further study.

Let $(\bar{M}, F, g)$ be a locally product Riemannian manifold and let $M$ be a submanifold of $\bar{M}$. A distribution $\mathcal{D}$ on $M$ is said to be a slant distribution if the angle $\theta$ between $F V$ and $\mathcal{D}_{p}$ is constant for $V \in \mathcal{D}_{p}$, i.e., it is independent of $p \in M$ and $V \in \mathcal{D}_{p}$. The constant angle $\theta$ is called the slant angle of the slant distribution $\mathcal{D}$. Thus, the invariant and anti-invariant distributions with respect to $F$ are slant distributions with slant angle $\theta=0$ and $\theta=\pi / 2$, respectively. A submanifold $M$ of $\bar{M}$ is said to be a slant submanifold if the tangent bundle $T M$ of $M$ is slant $[14,17]$. A slant submanifold that is neither invariant nor anti-invariant is called a proper slant submanifold.

Let $M$ be a slant submanifold with slant angle $\theta$ of a l.p.R. manifold $(\bar{M}, g, F)$, for any $V \in \Gamma(T M)$ and $\xi \in \Gamma\left(T^{\perp} M\right)$, we write

$$
F V=T V+N V \quad \text { and } \quad F \xi=t \xi+w \xi .
$$

Here $T V$ is the tangential part of $F V$ and $N V$ is the normal part of $F V$ also $t \xi$ is the tangential part of $F \xi$ and $w \xi$ is the normal part of $F \xi$. Then, using (2.10) and (3.1) we find

$$
T^{2}+t N=I, \quad N T+w N=0, \quad w^{2}+N t=I, \quad T t+t w=0 .
$$

Then, for any $U, V \in \Gamma(T M)$ we have [17]

$$
T^{2} V=\cos ^{2} \theta V
$$




$$
g(T U, T V)=\cos ^{2} \theta g(U, V) \quad \text { and } \quad g(N U, N V)=\sin ^{2} \theta g(U, V) .
$$

A submanifold $M$ of a l.p.R. manifold $(\bar{M}, F, g)$ is called a generalized semi-invariant submanifold if its tangent bundle $T M$ of $M$ has the form

$$
T M=\mathcal{D}^{\theta} \oplus \mathcal{D}_{1}^{T} \oplus \ldots \oplus \mathcal{D}_{k}^{T} \oplus \mathcal{D}_{1}^{\perp} \oplus \cdots \oplus \mathcal{D}_{l}^{\perp},
$$

where the distribution $\mathcal{D}_{\alpha}^{T}$ is an invariant for $1 \leq \alpha \leq k$, i.e., $F \mathcal{D}_{\alpha}^{T} \subseteq \mathcal{D}_{\alpha}^{T}$, the distribution $\mathcal{D}_{a}^{\perp}$ is an anti-invariant for $1 \leq a \leq l$, i.e. $F \mathcal{D}_{a}^{\perp} \subseteq T^{\perp} M$ and the distribution $\mathcal{D}^{\theta}$ is slant with slant angle $\theta$. In that case, the normal bundle $T^{\perp} M$ of $M$ decomposed as

$$
T^{\perp} M=N\left(\mathcal{D}^{\theta}\right) \oplus F\left(\mathcal{D}_{1}^{\perp}\right) \oplus \ldots \oplus F\left(\mathcal{D}_{l}^{\perp}\right) \oplus \overline{\mathcal{D}}^{T},
$$

where $\overline{\mathcal{D}}^{T}$ is the orthogonal complementary distribution of $N\left(\mathcal{D}^{\theta}\right) \oplus F\left(\mathcal{D}_{1}^{\perp}\right) \oplus \cdots \oplus F\left(\mathcal{D}_{l}^{\perp}\right)$ in $T^{\perp} M$ and it is invariant subbundle of $T^{\perp} M$ with respect to $F$. We say that a generalized semi-invariant submanifold is proper, neither $\theta=0$ nor $\theta=\frac{\theta}{2}$.

Remark 3.1. The notion of generalized semi-invariant submanifold of a 1.p.R. manifolds is a natural generalization of invariant, anti-invariant [1] semi-invariant [7], slant [17], semi-slant [15], hemi-slant [21] and skew semiinvariant submanifold of order 1 [20] of a 1.p.R. manifold. Also, this notion is slightly different from the definition of the skew semi-invariant submanifold [14]. For more details, we refer to [2, 4, 6, 24].

We need the following lemma.

Lemma 3.1. [20] Let $M$ be a generalized semi-invariant submanifold of a l.p.R. manifold $(\bar{M}, F, g)$. Then we have

$$
\begin{array}{r}
g\left(\nabla_{Z} W, U_{\alpha}\right)=-\csc ^{2} \theta\left\{g\left(A_{N T W} Z, U_{\alpha}\right)+g\left(A_{N W} Z, F U_{\alpha}\right)\right\}, \\
g\left(\nabla_{Z} W, X_{a}\right)=\sec ^{2} \theta\left\{g\left(A_{F X_{a}} Z, T W\right)+g\left(A_{N T W} Z, X_{a}\right)\right\}, \\
g\left(\nabla_{U_{\alpha}} V_{\alpha}, Z\right)=\csc ^{2} \theta\left\{g\left(A_{N T Z} U_{\alpha}, V_{\alpha}\right)+g\left(A_{N Z} U_{\alpha}, F V_{\alpha}\right)\right\}, \\
g\left(\nabla_{U_{\alpha}} V_{\alpha}, X_{a}\right)=g\left(A_{F X_{a}} U_{\alpha}, F V_{\alpha}\right), \\
g\left(\nabla_{X_{a}} Y_{a}, U_{\alpha}\right)=-g\left(A_{F Y_{a}} X_{a}, F U_{\alpha}\right), \\
g\left(\nabla_{X_{a}} Z, U_{\alpha}\right)=-\csc ^{2} \theta\left\{g\left(A_{N T Z} X_{a}, U_{\alpha}\right)+g\left(A_{N Z} X_{a}, F U_{\alpha}\right)\right\}, \\
g\left(\nabla_{Z} X_{a}, U_{\alpha}\right)=-g\left(A_{F X_{a}} Z, F U_{\alpha}\right), \\
g\left(\nabla_{U_{\alpha}} X_{a}, Z\right)=-\sec ^{2} \theta\left\{g\left(A_{F X_{a}} U_{\alpha}, T Z\right)+g\left(A_{N T Z} U_{\alpha}, X_{a}\right)\right\},
\end{array}
$$

for $U_{\alpha}, V_{\alpha} \in \Gamma\left(\mathcal{D}_{\alpha}^{T}\right)$ with $1 \leq \alpha \leq k, X_{a}, Y_{a} \in \Gamma\left(\mathcal{D}_{a}^{\perp}\right)$ with $1 \leq a \leq l$ and $Z, W \in \Gamma\left(\mathcal{D}^{\theta}\right)$.

Lemma 3.2. Let $M$ be a generalized semi-invariant submanifold of a l.p.R. manifold $(\bar{M}, F, g)$. Then we have

$$
\begin{gathered}
g\left(\nabla_{X_{a}} Y_{a}, Z\right)=-\sec ^{2} \theta\left\{g\left(A_{F Y_{a}} X_{a}, T Z\right)+g\left(A_{N T Z} X_{a}, Y_{a}\right)\right\}, \\
g\left(\nabla_{U_{\alpha}} V_{\alpha}, U_{\beta}\right)=g\left(\nabla_{U_{\alpha}} F V_{\alpha}, F U_{\beta}\right), \\
g\left(\nabla_{X_{a}} Y_{a}, X_{b}\right)=g\left(\nabla_{X_{a}}^{\perp} F Y_{a}, F X_{b}\right),
\end{gathered}
$$

for $U_{\alpha}, V_{\alpha} \in \Gamma\left(\mathcal{D}_{\alpha}^{T}\right), U_{\beta} \in \Gamma\left(\mathcal{D}_{\beta}^{T}\right)$ with $1 \leq \alpha \neq \beta \leq k, X_{a}, Y_{a} \in \Gamma\left(\mathcal{D}_{a}^{\perp}\right), X_{b} \in \Gamma\left(\mathcal{D}_{b}^{\perp}\right)$ with $1 \leq a \neq b \leq l$ and $Z \in$ $\Gamma\left(\mathcal{D}^{\theta}\right)$. 
Proof. Let $X_{a}, Y_{a} \in \Gamma\left(\mathcal{D}_{a}^{\perp}\right)$ and $Z \in \Gamma\left(\mathcal{D}^{\theta}\right)$. By using (2.5), (2.10) and (3.1), we have

$$
g\left(\nabla_{X_{a}} Y_{a}, Z\right)=g\left(\bar{\nabla}_{X_{a}} F Y_{a}, F Z\right)=g\left(\bar{\nabla}_{X_{a}} F Y_{a}, T Z\right)+g\left(\bar{\nabla}_{X_{a}} F Y_{a}, N Z\right) .
$$

Hence using (2.10) and (3.1) we have

$$
g\left(\nabla_{X_{a}} Y_{a}, Z\right)=-g\left(A_{F Y_{a}} X_{a}, T Z\right)+g\left(\bar{\nabla}_{X_{a}} Y_{a}, t N Z\right)+g\left(\bar{\nabla}_{X_{a}} Y_{a}, w N Z\right) .
$$

Again using (2.5), (3.3) and (3.2), we obtain

$$
g\left(\nabla_{X_{a}} Y_{a}, Z\right)=-g\left(A_{F Y_{a}} X_{a}, T Z\right)+\sin ^{2} \theta g\left(\bar{\nabla}_{X_{a}} Y_{a}, Z\right)-g\left(A_{N T Z} X_{a}, Y_{a}\right) .
$$

According to direct calculating we find (3.15). Let $U_{\alpha}, V_{\alpha} \in \Gamma\left(\mathcal{D}_{\alpha}^{T}\right), U_{\beta}, V_{\beta} \in \Gamma\left(\mathcal{D}_{\beta}^{T}\right)$. Then using (2.5), we have $g\left(\nabla_{U_{\alpha}} V_{\alpha}, U_{\beta}\right)=g\left(\bar{\nabla}_{U_{\alpha}} V_{\alpha}, U_{\beta}\right)$. By using (2.10), we obtain $g\left(\nabla_{U_{\alpha}} V_{\alpha}, U_{\beta}\right)=g\left(F \bar{\nabla}_{U_{\alpha}} V_{\alpha}, F U_{\beta}\right)$. Hence using (2.11), we get $g\left(\nabla_{U_{\alpha}} V_{\alpha}, U_{\beta}\right)=g\left(\bar{\nabla}_{U_{\alpha}} F V_{\alpha}, F U_{\beta}\right)$, since $F U_{\beta} \in \Gamma(T M)$. With the help of (2.5) we obtain (3.16)

$$
g\left(\nabla_{U_{\alpha}} V_{\alpha}, U_{\beta}\right)=g\left(\nabla_{U_{\alpha}} F V_{\alpha}, F U_{\beta}\right) .
$$

Let $X_{a}, Y_{a} \in \Gamma\left(\mathcal{D}_{a}^{\perp}\right), X_{b}, Y_{b} \in \Gamma\left(\mathcal{D}_{b}^{\perp}\right)$. Then using (2.5) we have $g\left(\nabla_{X_{a}} Y_{a}, X_{b}\right)=g\left(\bar{\nabla}_{X_{a}} Y_{a}, X_{b}\right)$. By using (2.10), we obtain $g\left(\nabla_{X_{a}} Y_{a}, X_{b}\right)=g\left(F \bar{\nabla}_{U_{\alpha}} V_{\alpha}, F X_{b}\right)$. Hence by using (2.11), we get $g\left(\nabla_{X_{a}} Y_{a}, X_{b}\right)=g\left(\bar{\nabla}_{X_{a}}^{\perp} F Y_{a}, F X_{b}\right)$, since $F X_{b} \in \Gamma\left(T M^{\perp}\right)$. With the help of (2.5) we obtain (3.16) $g\left(\nabla_{X_{a}} Y_{a}, X_{b}\right)=g\left(\nabla_{X_{a}}^{\perp} F Y_{a}, F X_{b}\right)$.

Theorem 3.1. Let $M$ be a generalized proper semi-invariant submanifold of a l.p.R manifold $(\bar{M}, F, g)$. Then the slant distribution $D^{\theta}$ is totally geodesic if and only if the following equations hold

$$
\begin{aligned}
& g\left(A_{N T W} Z, U_{\alpha}\right)=-g\left(A_{N W} Z, F U_{\alpha}\right), \\
& g\left(A_{F X_{a}} Z, T W\right)=-g\left(A_{N T W} Z, X_{a}\right)
\end{aligned}
$$

for $Z, W \in \Gamma\left(\mathcal{D}^{\theta}\right), U_{\alpha} \in \Gamma\left(\mathcal{D}_{\alpha}^{T}\right)$ and $X_{a} \in \Gamma\left(\mathcal{D}_{a}^{\perp}\right)$.

Proof. Let $M$ be a generalized semi-invariant submanifold of a 1.p.R manifold $(\bar{M}, F, g)$. Then the slant distribution $D^{\theta}$ is totally geodesic if and only if $g\left(\nabla_{Z} W, X_{a}\right)=0$ and $g\left(\nabla_{Z} W, U_{\alpha}\right)=0$ for all $Z, W \in \Gamma\left(\mathcal{D}^{\theta}\right)$, $X_{a} \in \Gamma\left(\mathcal{D}_{a}^{\perp}\right)$ and $U_{\alpha} \in \Gamma\left(\mathcal{D}_{\alpha}^{T}\right)$. Thus, the assertions (3.18) and (3.19) follow from (3.7) and (3.8), respectively.

Theorem 3.2. Let $M$ be a generalized proper semi-invariant submanifold of a l.p.R manifold $(\bar{M}, F, g)$. Then the invariant distribution $D_{\alpha}^{T}, 1 \leq \alpha \leq k$ is integrable if and only if the following equations hold

$$
\begin{aligned}
g\left(A_{F X_{\alpha}} U_{\alpha}, F V_{\alpha}\right) & =g\left(A_{F X_{\alpha}} V_{\alpha}, F U_{\alpha}\right), \\
g\left(A_{N T Z} U_{\alpha}, V_{\alpha}\right)+g\left(A_{N Z} U_{\alpha}, F V_{\alpha}\right) & =g\left(A_{N T Z} V_{\alpha}, U_{\alpha}\right)+g\left(A_{N Z} V_{\alpha}, F U_{\alpha}\right), \\
g\left(\nabla_{U_{\alpha}} F V_{\alpha}, F U_{\beta}\right) & =g\left(\nabla_{V_{\alpha}} F U_{\alpha}, F U_{\beta}\right),
\end{aligned}
$$

for $Z \in \Gamma\left(\mathcal{D}^{\theta}\right), U_{\alpha}, V_{\alpha} \in \Gamma\left(\mathcal{D}_{\alpha}^{T}\right), U_{\beta} \in \Gamma\left(\mathcal{D}_{\beta}^{T}\right), 1 \leq \alpha \neq \beta \leq k$ and $X_{a} \in \Gamma\left(\mathcal{D}_{a}^{\perp}\right)$.

Proof. Let $M$ be a generalized semi-invariant submanifold of a l.p.R manifold $(\bar{M}, F, g)$. Then the invariant distribution $D_{\alpha}^{T}$ is integrable if and only if $g\left(\left[U_{\alpha}, V_{\alpha}\right], X_{a}\right)=0, g\left(\left[U_{\alpha}, V_{\alpha}\right], Z\right)=0$ and $g\left(\left[U_{\alpha}, V_{\alpha}\right], U_{\beta}\right)=0$ for all $Z \in \Gamma\left(\mathcal{D}^{\theta}\right), X_{a} \in \Gamma\left(\mathcal{D}_{a}^{\perp}\right)$ and $U_{\alpha}, V_{\alpha} \in \Gamma\left(\mathcal{D}_{\alpha}^{T}\right), U_{\beta} \in \Gamma\left(\mathcal{D}_{\beta}^{T}\right)$ with $1 \leq \alpha \neq \beta \leq k$. Thus, the assertions (3.20), (3.21) and (3.22) follow from (3.9), (3.10) and (3.16), respectively.

Theorem 3.3. Let $M$ be a generalized proper semi-invariant submanifold of a l.p.R manifold $(\bar{M}, F, g)$. Then the antiinvariant distribution $D_{a}^{\perp}, 1 \leq a \leq l$ is integrable if and only if the following equations hold

$$
\begin{gathered}
g\left(A_{F X_{a}} Y_{a}, F U_{\alpha}\right)=g\left(A_{F Y_{a}} X_{a}, F U_{\alpha}\right), \\
g\left(A_{F Y_{a}} X_{a}, T Z\right)=g\left(A_{F X_{a}} Y_{a}, T Z\right), \\
g\left(\nabla_{X_{a}}^{\perp} F Y_{a}, X_{b}\right)=g\left(\nabla_{Y_{a}}^{\perp} F X_{a}, X_{b}\right),
\end{gathered}
$$

for $Z \in \Gamma\left(\mathcal{D}^{\theta}\right), U_{\alpha} \in \Gamma\left(\mathcal{D}_{\alpha}^{T}\right)$ and $X_{a}, Y_{a} \in \Gamma\left(\mathcal{D}_{a}^{\perp}\right), X_{b} \in \Gamma\left(\mathcal{D}_{b}^{\perp}\right), 1 \leq a \neq b \leq l$.

Proof. Let $M$ be a generalized semi-invariant submanifold of a l.p.R manifold $(\bar{M}, F, g)$. Then the anti-invariant distribution $D_{a}^{\perp}$ is integrable if and only if $g\left(\left[X_{a}, Y_{a}\right], Z\right)=0, g\left(\left[X_{a}, Y_{a}\right], U_{\alpha}\right)=0$ and $g\left(\left[X_{a}, Y_{a}\right], X_{b}\right)=0$ for all $Z \in \Gamma\left(\mathcal{D}^{\theta}\right), U_{\alpha} \in \Gamma\left(\mathcal{D}_{\alpha}^{T}\right)$ and $X_{a}, Y_{a} \in \Gamma\left(\mathcal{D}_{a}^{\perp}\right), X_{b} \in \Gamma\left(\mathcal{D}_{b}^{\perp}\right)$ with $1 \leq a \neq b \leq l$. Thus, the assertions (3.23) and (3.25) follow from (3.11), (3.15) and (3.17), respectively. 


\section{Certain Types of Multiply Warped Product Submanifolds in Locally Product Riemannian Manifolds}

In this section, we check that the existence of certain types of multiply warped product generalized semi-invariant submanifolds in the form,

$$
\begin{aligned}
& \text { I. } M^{T} \times_{\sigma_{1}} M_{1}^{\perp} \times \ldots \times_{\sigma_{l}} M_{1}^{\perp} \times_{\lambda_{1}} M_{1}^{\theta_{1}} \times \ldots \times_{\lambda_{m}} M_{m}^{\theta_{m}}, \\
& \text { II. } M^{\perp} \times_{f_{1}} M_{1}^{T} \times \ldots \times_{f_{k}} M_{k}^{T} \times_{\lambda_{1}} M^{\theta_{1}} \times \ldots \times_{\lambda_{m}} M_{m}^{\theta_{m}} \text {, } \\
& \text { III. } \quad M^{\theta} \times_{f_{1}} M_{1}^{T} \times \ldots \times_{f_{k}} M_{k}^{T} \times_{\sigma_{1}} M_{1}^{\perp} \times \ldots \times_{\sigma_{l}} M_{l}^{\perp} \text {, }
\end{aligned}
$$

where $M_{\alpha}^{T}, 1 \leq \alpha \leq k$ is an invariant, $M_{a}^{\perp}, 1 \leq a \leq l$ is an anti-invariant and $M_{\beta}^{\theta_{\beta}}$ is a proper slant submanifold with slant angle $\theta_{\beta}, 1 \leq \beta \leq m$ of a l.p.R manifold $(\bar{M}, F, g)$.

M. Atçeken and B. Sahin independently proved that there do not exist (non-trivial) warped product semiinvariant submanifolds in the form $M^{T} \times_{f} M^{\perp}$ in a 1.p.R. manifold $(\bar{M}, F, g)$, such that $M^{T}$ is an invariant submanifold and $M_{\perp}$ is an anti-invariant submanifold of $(\bar{M}, F, g)$ in Theorem 3.1([5]) and Theorem 3.1([19]), respectively. Again, M. Atçeken and B. Șahin independently proved that there do not exist (non-trivial) warped product semi-slant submanifolds in the form $M^{T} \times_{f} M^{\theta}$ in a l.p.R. manifold $\bar{M}$, such that $M^{T}$ is an invariant submanifold and $M^{\theta}$ is a proper slant submanifold of $\bar{M}$ in Theorem 3.3([3]) and Theorem 3.1([18]), respectively. Thus, we obtain the following result.

Corollary 4.1. There do not exist (non-trivial) multiply warped product generalized semi-invariant submanifold in the form I of a l.p.R. manifold $(\bar{M}, F, g)$.

On the other hand, it was proved that there do not exist (non-trivial) warped product semi-invariant submanifold in the form $M^{\perp} \times_{f} M^{\theta}$ in a l.p.R. manifold $\bar{M}$ such that $M^{\perp}$ is an anti-invariant submanifold and $M^{\theta}$ is a proper slant submanifold of $\bar{M}$ in Theorem 3.4 of [3]. Thus, we deduce the following result.

Corollary 4.2. There do not exist (non-trivial) multiply warped product generalized semi-invariant submanifold in the form II of a l.p.R. manifold $(\bar{M}, F, g)$.

Now, we consider (non-trivial) multiply warped product generalized semi-invariant submanifolds in the form $M^{\theta} \times_{f_{1}} M_{1}^{T} \times \ldots \times_{f_{k}} M_{k}^{T} \times_{\sigma_{1}} M_{1}^{\perp} \times \ldots \times_{\sigma_{l}} M_{l}^{\perp}$ in a 1.p.R. manifold $(\bar{M}, F, g)$ such that $M_{\alpha}^{T}, 1 \leq \alpha \leq k$ is an invariant, $M_{a}^{\perp}, 1 \leq a \leq l$ is an anti-invariant and $M^{\theta}$ is a proper slant submanifold of $\bar{M}$. We first present an example of such a submanifold.

Example 4.1. Consider the $4 k+4 l$-dimensional Euclidean space $R^{4 k+4 l}$ with usual metric $g$ and almost product structure $F$ defined by

$$
\begin{gathered}
F \partial_{i}=\partial_{i}, \quad 1 \leq i \leq 2 k, \quad F \partial_{i}=-\partial_{i}, \quad 2 k+1 \leq i \leq 4 k, \\
F \partial_{j}=\partial_{j+1}, \quad F \partial_{j+1}=\partial_{j} \quad 4 k+1 \leq j \leq 4 k+4 l,
\end{gathered}
$$

where $\partial_{s}=\frac{\partial}{\partial x_{s}}$ and $\left\{x_{s}\right\}_{1 \leq s \leq 4 k+4 l}$ are natural coordinates of $R^{4 k+4 l}$. Upon straightforward calculation, we see that $\left(R^{4 k+4 l}, F, g\right)$ is a l.p.R. manifold. Let $M$ be a submanifold of $\left(R^{4 k+4 l}, F, g\right)$ given by

$$
\begin{aligned}
& x_{1}=t \sin u_{1}, \quad x_{2}=t \cos u_{1}, \\
& x_{3}=2 t \sin u_{2}, \quad x_{4}=2 t \cos u_{2}, \\
& \begin{array}{c}
\cdots \\
x_{2 k-1}=k t \sin u_{k}, \quad x_{2 k}=k t \cos u_{k},
\end{array} \\
& x_{2 k+1}=\frac{t}{\sqrt{2}} \cos v_{1}, x_{2 k+2}=\frac{t}{\sqrt{2}} \sin v_{1} \text {, } \\
& x_{2 k+3}=\frac{2 t}{\sqrt{2}} \cos v_{2}, x_{2 k+4}=\frac{2 t}{\sqrt{2}} \sin v_{2} \text {, } \\
& \text {.. } \ldots \text {, }
\end{aligned}
$$




$$
\begin{aligned}
x_{4 k-1}=\frac{k t}{\sqrt{2}} \cos v_{k}, & x_{4 k}=\frac{k t}{\sqrt{2}} \sin v_{k}, \\
x_{4 k+1}=2 t \sin z_{1}, & x_{4 k+2}=0, \\
x_{4 k+3}=2 t \cos z_{1}, & x_{4 k+4}=0, \\
x_{4 k+5}=2 t \sin z_{2}, & x_{4 k+6}=0, \\
x_{4 k+7}=2 t \cos z_{2}, & x_{4 k+8}=0, \\
\ldots & \ldots, \\
x_{4 k+4 l-3}=2 l t \sin z_{l}, & x_{4 k+4 l-2}=0, \\
x_{4 k+4 l-1}=2 l t \cos z_{l}, & x_{4 k+4 l}=0 .
\end{aligned}
$$

where $u_{i}, v_{i}, z_{j} \in\left(0, \frac{\pi}{2}\right)$ and $t>0$. Then, the local frame of $T M$ given by

$$
\begin{aligned}
T= & \sin u_{1} \partial_{1}+\cos u_{1} \partial_{2}+2 \sin u_{2} \partial_{3}+2 \cos u_{2} \partial_{4}+\cdots+k \sin u_{k} \partial_{2 k-1} \\
& +k \cos u_{k} \partial_{2 k} \\
& +\frac{1}{\sqrt{2}}\left\{\cos v_{1} \partial_{2 k+1}+\sin v_{1} \partial_{2 k+2}+2 \cos v_{2} \partial_{2 k+3}+2 \sin v_{2} \partial_{2 k+4}\right. \\
& \left.+\cdots+k \cos v_{k} \partial_{4 k-1}+k \sin v_{k} \partial_{4 k}\right\} \\
& +2\left\{\sin z_{1} \partial_{4 k+1}+\cos z_{1} \partial_{4 k+3}+2 \sin z_{2} \partial_{4 k+5}+2 \cos z_{2} \partial_{4 k+7}\right. \\
& \left.+\cdots+l \sin z_{l} \partial_{4 k+4 l-3}+l \cos z_{l} \partial_{4 k+4 l-1}\right\}
\end{aligned}
$$

$$
\begin{aligned}
& U_{1}=t \cos u_{1} \partial_{1}-t \sin u_{1} \partial_{2}, \\
& U_{2}=2 t \cos u_{2} \partial_{3}-2 t \sin u_{2} \partial_{4} \text {, } \\
& U_{k}=k t \cos u_{k} \partial_{2 k-1}-k t \sin u_{k} \partial_{2 k}, \\
& V_{1}=-\frac{t}{\sqrt{2}} \sin v_{1} \partial_{2 k+1}+\frac{t}{\sqrt{2}} \cos v_{1} \partial_{2 k+2}, \\
& V_{2}=-\frac{2 t}{\sqrt{2}} \sin v_{2} \partial_{2 k+3}+\frac{2 t}{\sqrt{2}} \cos v_{2} \partial_{2 k+4} \text {, } \\
& V_{k}=-\frac{k t}{\sqrt{2}} \sin v_{k} \partial_{4 k-1}+\frac{k t}{\sqrt{2}} \cos v_{k} \partial_{4 k}, \\
& Z_{1}=2 t \cos z_{1} \partial_{4 k+1}-2 t \sin z_{1} \partial_{4 k+3} \text {, } \\
& Z_{2}=2 t \cos z_{2} \partial_{4 k+5}-2 t \sin z_{2} \partial_{4 k+7} \text {, } \\
& Z_{l}=2 l t \cos z_{l} \partial_{4 k+4 l-3}-2 l t \sin z_{l} \partial_{4 k+4 l-1} .
\end{aligned}
$$

By direct calculation, we see that $\mathcal{D}^{\theta}=\operatorname{span}\{T\}$ is a proper slant distribution with slant angle $\theta=\cos ^{-1}\left(\frac{1}{3}+\right.$ $\left.\frac{k(k+1)(2 k+1)}{8 l(l+1)(2 l+1)}\right)$ and $\mathcal{D}_{i}^{T}=\operatorname{span}\left\{U_{i}, V_{i}\right\}, 1 \leq i \leq k$ is an invariant distribution and $\mathcal{D}_{j}^{\perp}=\operatorname{span}\left\{Z_{j}\right\}, 1 \leq j \leq l$ is an anti-invariant distribution. So far, $M$ is a proper generalized semi-invariant submanifold. Moreover, $\mathcal{D}^{\theta}$ is totally geodesic and both $\mathcal{D}_{i}^{T}$ and $\mathcal{D}_{j}^{\perp}$ are integrables distributions. If we denote the integral manifolds of $\mathcal{D}^{\theta}$, $\mathcal{D}_{i}^{T}$ and $\mathcal{D}_{j}^{\perp}$ by $M^{\theta}, M_{i}^{T}$ and $M_{j}^{\perp}$, respectively, then the induced metric tensor of $M$ is

$$
d s^{2}=g(T, T) d t^{2}+\sum_{i=1}^{k} g\left(U_{i}, U_{i}\right) d u_{i}^{2}+\sum_{i=1}^{k} g\left(V_{i}, V_{i}\right) d u_{j}^{2}+\sum_{j=1}^{l} g\left(Z_{j}, Z_{j}\right) d z_{j}^{2} .
$$

Upon straightforward calculation, we have

$$
\begin{aligned}
d s^{2}= & \frac{1}{12}[3 k(k+1)(2 k+1)+8 l(l+1)(2 l+1)] d t^{2}+t^{2}\left(d u_{1}^{2}+\frac{1}{2} d v_{1}^{2}\right)+ \\
& (2 t)^{2}\left(d u_{2}^{2}+\frac{1}{2} d v_{2}^{2}\right)+\ldots+(k t)^{2}\left(d u_{k}^{2}+\frac{1}{2} d v_{k}^{2}\right)+(2 t)^{2} d z_{1}^{2}+ \\
& (4 t)^{2} d z_{2}^{2}+\ldots+(2 l t)^{2} d z_{l}^{2} \\
= & g_{M^{\theta}}+t^{2} g_{M_{1}^{T}}+(2 t)^{2} g_{M_{2}^{T}}+\ldots+(k t)^{2} g_{M_{k}^{T}}+(2 t)^{2} g_{M_{1}^{\perp}}+(4 t)^{2} g_{M_{2}^{\perp}}+ \\
& \ldots+(2 l t)^{2} g_{M_{l}}
\end{aligned}
$$


Thus, $M=M^{\theta} \times_{f_{1}} M_{1}^{T} \times \ldots \times_{f_{k}} M_{k}^{T} \times_{\sigma_{1}} M_{1}^{\perp} \times \ldots \times_{\sigma_{l}} M_{l}^{\perp}$ is a (non-trivial) multiply warped product generalized semi-invariant submanifold of $\left(R^{4 k+4 l}, F, g\right)$ with warping functions $f_{1}=t, f_{2}=2 t, \ldots, f_{k}=k t$ and $\sigma_{1}=2 t, \sigma_{2}=4 t, \ldots, \sigma_{l}=2 l t$.

\section{Multiply warped product generalized semi-invariant submanifolds}

In this section, we give a characterization for a multiply warped product proper generalized semiinvariant submanifold in the form $M^{\theta} \times_{f_{1}} M_{1}^{T} \times \ldots \times_{f_{k}} M_{k}^{T} \times_{\sigma_{1}} M_{1}^{\perp} \times \ldots \times_{\sigma_{l}} M_{l}^{\perp}$, where $M^{\theta}$ is a proper slant submanifold, $M_{\alpha}^{T}, 1 \leq \alpha \leq k$ is an invariant and $M_{a}^{\perp}, 1 \leq a \leq l$ is an anti invariant submanifold of a 1.p.R. manifold $(\bar{M}, F, g)$. After that we investigate the behavior of the second fundemental form of such submanifolds and as a result, we give a necessary and sufficient condition for such submanifolds to be locally multiply warped product generalized. We first recall the following fact given in [12] to prove our theorem.

Remark 5.1. (Remark 2.1 [12]) Suppose that the tangent bundle of a Riemannian manifold $M$ splints into an orthogonal sum $T M=\mathcal{D}_{0} \oplus \mathcal{D}_{1} \oplus \ldots \oplus \mathcal{D}_{k}$ of non-trivial distributions such that each $\mathcal{D}_{j}$ is spherical and its complement in $T M$ is autoparallel for $j \in\{1,2, \ldots, k\}$. Then the manifold $M$ is locally isometric to a multiply warped product $M_{0} \times_{f_{1}} M_{1} \times \ldots \times{ }_{f_{k}} M_{k}$.

Now, we give one of the main theorems of this paper.

Theorem 5.1. Let $M$ be $a\left(\mathcal{D}^{\theta}, \mathcal{D}_{a}^{\perp}\right)$-mixed geodesic multiply warped product generalized semi-invariant submanifold of a l.p.R. manifold $(\bar{M}, F, g)$. Then $M$ is a locally multiply warped product generalized submanifold of type $M^{\theta} \times_{f_{1}} M_{1}^{T} \times$ $\ldots \times_{f_{k}} M_{k}^{T} \times_{\sigma_{1}} M_{1}^{\perp} \times \ldots \times_{\sigma_{l}} M_{l}^{\perp}$ if and only if we have

$$
\begin{gathered}
A_{N T Z} X_{a}=\cos ^{2} \theta Z(\lambda) X_{a}, \\
A_{N Z} U_{\alpha}+A_{N T Z} F U_{\alpha}=-\sin ^{2} \theta Z(\mu) F U_{\alpha},
\end{gathered}
$$

for some functions $\lambda$ and $\mu$ satisfying $X_{a}(\lambda)=U_{\alpha}(\lambda)=0$ and $X_{a}(\mu)=U_{\alpha}(\mu)=0$

$$
\begin{gathered}
g\left(A_{F X_{a}} Z, T W\right)=-g\left(A_{N T W} Z, X_{a}\right), \\
g\left(A_{F X_{a}} U_{\alpha}, F V_{\alpha}\right)=0, \\
g\left(A_{F Y_{a}} X_{a}, F V_{\alpha}\right)=0, \\
g\left(A_{F X_{a}} Z, F U_{\alpha}\right)=0, \\
g\left(A_{F X_{a}} U_{\alpha}, T Z\right)=-g\left(A_{N T Z} U_{\alpha}, X_{a}\right), \\
g\left(\nabla_{U_{\beta}} U_{\gamma}, U_{\alpha}\right)=0, \\
g\left(\nabla_{X_{b}} X_{c}, X_{a}\right)=0
\end{gathered}
$$

and (3.22) and (3.25) hold, where $Z, W \in \Gamma\left(\mathcal{D}^{\theta}\right), U_{\alpha}, V_{\alpha} \in \Gamma\left(\mathcal{D}_{\alpha}^{T}\right), U_{\beta} \in \Gamma\left(\mathcal{D}_{\beta}^{T}\right)$ and $U_{\gamma} \in \Gamma\left(\mathcal{D}_{\gamma}^{T}\right)$ for $1 \leq \alpha, \beta, \gamma \leq k$ with $\alpha \neq \beta$ and $\alpha \neq \gamma, X_{a}, Y_{a} \in \Gamma\left(\mathcal{D}_{a}^{\perp}\right), X_{b} \in \Gamma\left(\mathcal{D}_{b}^{\perp}\right)$ and $X_{c} \in \Gamma\left(\mathcal{D}_{c}^{\perp}\right)$ for $1 \leq a, b, c \leq l$ with $a \neq b$ and $a \neq c$.

Proof. Let $M$ be a $\left(\mathcal{D}^{\theta}, \mathcal{D}_{a}^{\perp}\right)$-mixed geodesic multiply warped product generalized semi-invariant submanifold of a 1.p.R. manifold $(\bar{M}, F, g)$ in the form $M^{\theta} \times_{f_{1}} M_{1}^{T} \times \ldots \times_{f_{k}} M_{k}^{T} \times_{\sigma_{1}} M_{1}^{\perp} \times \ldots \times_{\sigma_{l}} M_{l}^{\perp}$. Since $M$ is $\left(\mathcal{D}^{\theta}, \mathcal{D}_{a}^{\perp}\right)$ mixed geodesic, for $Z, W \in \Gamma\left(\mathcal{D}^{\theta}\right)$ and $X_{a} \in \Gamma\left(\mathcal{D}_{a}^{\perp}\right)$ with $1 \leq a \leq l$, using (2.6), we find

$$
g\left(A_{N T Z} X_{\alpha}, W\right)=g\left(h\left(X_{\alpha}, W\right), N T Z\right)=0 .
$$

Moreover for any $U_{\alpha} \in \Gamma\left(\mathcal{D}_{\alpha}^{T}\right)$ with $1 \leq \alpha \leq k$, using (2.5) and (3.1),

$$
g\left(A_{N T Z} X_{a}, U_{\alpha}\right)=-g\left(\bar{\nabla}_{X_{a}} N T Z, U_{\alpha}\right)=-g\left(\bar{\nabla}_{X_{a}} F T Z, U_{\alpha}\right)+g\left(\bar{\nabla}_{X_{a}} T^{2} Z, U_{\alpha}\right) .
$$

Then using (2.9) (2.11) and (3.3), we find

$$
g\left(A_{N T Z} X_{a}, U_{\alpha}\right)=-g\left(\bar{\nabla}_{X_{a}} T Z, F U_{\alpha}\right)+\cos ^{2} \theta g\left(\bar{\nabla}_{X_{a}} Z, U_{\alpha}\right) .
$$

Here, using (2.5), we arrive

$$
g\left(A_{N T Z} X_{a}, U_{\alpha}\right)=-g\left(\nabla_{X_{a}} T Z, F U_{\alpha}\right)+\cos ^{2} \theta g\left(\nabla_{X_{a}} Z, U_{\alpha}\right) .
$$


So, using (2.3), we conclude

$$
g\left(A_{N T Z} X_{a}, U_{\alpha}\right)=-T Z\left(\ln \sigma_{a}\right) g\left(X_{a}, F U_{\alpha}\right)+\cos ^{2} \theta Z\left(\ln \sigma_{a}\right) g\left(X_{a}, U_{\alpha}\right)=0 .
$$

Next, by a similar argument, for $Y_{a} \in \Gamma\left(\mathcal{D}_{a}^{\perp}\right)$, using (2.5) and (3.1) we have

$$
g\left(h\left(X_{a}, Y_{a}\right), N Z\right)=g\left(\bar{\nabla}_{X_{a}} Y_{a}, N Z\right)=g\left(\bar{\nabla}_{X_{a}} Y_{a}, F Z\right)-g\left(\bar{\nabla}_{X_{a}} Y_{a}, T Z\right) .
$$

Then using (2.10),(2.11) and (2.3), we find

$$
g\left(h\left(X_{a}, Y_{a}\right), N Z\right)=g\left(\bar{\nabla}_{X_{a}} F Y_{a}, Z\right)+T Z\left(\ln \sigma_{a}\right) g\left(X_{a}, Y_{a}\right) .
$$

Hence using (2.5) and (2.6), we arrive

In this equation, if $T Z$ is written instead of $Z$, we have

$$
\begin{aligned}
& g\left(h\left(X_{a}, Y_{a}\right), N Z\right)=-g\left(A_{F Y_{a}} X_{a}, Z\right)+T Z\left(\ln \sigma_{a}\right) g\left(X_{a}, Y_{a}\right) \\
& =-g\left(h\left(X_{a}, Z\right), F Y_{a}\right)+T Z\left(\ln \sigma_{a}\right) g\left(X_{a}, Y_{a}\right) .
\end{aligned}
$$

$$
g\left(h\left(X_{a}, Y_{a}\right), N T Z\right)=-g\left(h\left(X_{a}, T Z\right), F Y_{a}\right)+\cos ^{2} \theta Z\left(\ln \sigma_{a}\right) g\left(X_{a}, Y_{a}\right) .
$$

Since $M$ is $\left(\mathcal{D}^{\theta}, \mathcal{D}_{a}^{\perp}\right)$-mixed geodesic, we conclude that

$$
g\left(A_{N T Z} X_{a}, Y_{a}\right)=\cos ^{2} \theta Z\left(\ln \sigma_{a}\right) g\left(X_{a}, Y_{a}\right) .
$$

Moreover, we have $X_{a}\left(\ln \sigma_{a}\right)=U_{\alpha}\left(\ln \sigma_{a}\right)=0$, since $\sigma_{a}$ depends on only points of $M_{\theta}$. So, we conclude that $\lambda=\ln \sigma_{a}$. Thus, from (5.10) (5.12), it follows that (5.1). Now, we prove (5.2). For $Z \in \Gamma\left(\mathcal{D}^{\theta}\right), U_{\alpha} \in \Gamma\left(\mathcal{D}_{\alpha}^{T}\right)$ and $X_{a} \in \Gamma\left(\mathcal{D}_{a}^{\perp}\right)$, using (2.5) and (3.1), we have

$$
\begin{aligned}
g\left(A_{N Z} U_{\alpha}+A_{N T Z} F U_{\alpha}, X_{a}\right)= & g\left(A_{N Z} U_{\alpha}, X_{a}\right)+g\left(A_{N T Z} F U_{\alpha}, X_{a}\right) \\
= & g\left(A_{N Z} X_{a}, U_{\alpha}\right)+g\left(A_{N T Z} X_{a}, F U_{\alpha}\right) \\
= & -g\left(\bar{\nabla}_{X_{a}} N Z, U_{\alpha}\right)-g\left(\bar{\nabla}_{X_{a}} N T Z, F U_{\alpha}\right) \\
= & -g\left(\bar{\nabla}_{X_{a}} N Z, U_{\alpha}\right)-g\left(\bar{\nabla}_{X_{a}} F T Z, F U_{\alpha}\right) \\
& +g\left(\bar{\nabla}_{X_{a}} T^{2} Z, F U_{\alpha}\right) .
\end{aligned}
$$

Using (2.10), (2.11), (3.1) and (3.3) and, we arrive

$$
\begin{aligned}
g\left(A_{N Z} U_{\alpha}+A_{N T Z} F U_{\alpha}, X_{a}\right)= & -g\left(\bar{\nabla}_{X_{a}} F Z, U_{\alpha}\right)+g\left(\bar{\nabla}_{X_{a}} T Z, U_{\alpha}\right)-g\left(\bar{\nabla}_{X_{a}} T Z, U_{\alpha}\right) \\
& +\cos ^{2} \theta g\left(\bar{\nabla}_{X_{a}} Z, F U_{\alpha}\right) \\
= & -g\left(\bar{\nabla}_{X_{a}} F Z, U_{\alpha}\right)+\cos ^{2} \theta g\left(\bar{\nabla}_{X_{a}} Z, F U_{\alpha}\right) .
\end{aligned}
$$

Then, using (2.3), (2.5), (2.9) (2.11), we find

$$
\begin{aligned}
g\left(A_{N Z} U_{\alpha}+A_{N T Z} F U_{\alpha}, X_{a}\right) & =-g\left(\bar{\nabla}_{X_{a}} Z, F U_{\alpha}\right)+\cos ^{2} \theta g\left(\nabla_{X_{a}} Z, F U_{\alpha}\right) \\
& =-\sin ^{2} \theta g\left(\nabla_{X_{a}} Z, F U_{\alpha}\right) \\
& =-\sin ^{2} \theta Z\left(\ln \sigma_{a}\right) g\left(X_{a}, F U_{\alpha}\right) .
\end{aligned}
$$

Since $g\left(X_{a}, F U_{\alpha}\right)=0$, we conclude that

$$
g\left(A_{N Z} U_{\alpha}+A_{N T Z} F U_{\alpha}, X_{a}\right)=-\sin ^{2} \theta Z\left(\ln \sigma_{a}\right) g\left(X_{a}, F U_{\alpha}\right)=0 .
$$

Similarly, for $Z, W \in \Gamma\left(\mathcal{D}^{\theta}\right)$ and $U_{\alpha} \in \Gamma\left(\mathcal{D}_{\alpha}^{T}\right)$, using (2.5) and (3.1), we have

$$
\begin{aligned}
g\left(A_{N Z} U_{\alpha}+A_{N T Z} F U_{\alpha}, W\right)= & g\left(A_{N Z} U_{\alpha}, W\right)+g\left(A_{N T Z} F U_{\alpha}, W\right) \\
= & g\left(A_{N Z} W, U_{\alpha}\right)+g\left(A_{N T Z} W, F U_{\alpha}\right) \\
= & -g\left(\bar{\nabla}_{W} N Z, U_{\alpha}\right)-g\left(\bar{\nabla}_{W} N T Z, F U_{\alpha}\right) \\
= & -g\left(\bar{\nabla}_{W} N Z, U_{\alpha}\right)-g\left(\bar{\nabla}_{W} F T Z, F U_{\alpha}\right) \\
& +g\left(\bar{\nabla}_{W} T^{2} Z, F U_{\alpha}\right) .
\end{aligned}
$$

Using (2.10), (2.11), (3.1) and (3.3), we arrive

$$
\begin{aligned}
g\left(A_{N Z} U_{\alpha}+A_{N T Z} F U_{\alpha}, W\right)= & -g\left(\bar{\nabla}_{W} F Z, U_{\alpha}\right)+g\left(\bar{\nabla}_{W} T Z, U_{\alpha}\right)-g\left(\bar{\nabla}_{W} T Z, U_{\alpha}\right) \\
& +\cos ^{2} \theta g\left(\bar{\nabla}_{W} Z, F U_{\alpha}\right) \\
= & -g\left(\bar{\nabla}_{W} F Z, U\right)+\cos ^{2} \theta g\left(\bar{\nabla}_{W} Z, F U_{\alpha}\right) .
\end{aligned}
$$

Then, using (2.3), (2.5), (2.9) (2.11), we find

$$
\begin{aligned}
g\left(A_{N Z} U_{\alpha}+A_{N T Z} F U_{\alpha}, W\right) & =-g\left(\bar{\nabla}_{W} Z, F U_{\alpha}\right)+\cos ^{2} \theta g\left(\nabla_{W} Z, F U_{\alpha}\right) \\
& =-g\left(\nabla_{W} Z, F U_{\alpha}\right)+\cos ^{2} \theta g\left(\nabla_{W} Z, F U_{\alpha}\right) \\
& =-\sin ^{2} \theta g\left(\nabla_{W} Z, F U_{\alpha}\right)=+\sin ^{2} \theta g\left(Z, \nabla_{W}^{\theta} F U_{\alpha}\right) .
\end{aligned}
$$


Since $g\left(Z, \nabla_{W}^{\theta} F U_{\alpha}\right)=0$, we conclude

$$
g\left(A_{N Z} U_{\alpha}+A_{N T Z} F U_{\alpha}, W\right)=\sin ^{2} \theta g\left(Z, \nabla_{W}^{\theta} F U_{\alpha}\right)=0 .
$$

On the other hand, for $Z \in \Gamma\left(\mathcal{D}^{\theta}\right)$ and $U_{\alpha}, V_{\alpha} \in \Gamma\left(\mathcal{D}_{\alpha}^{T}\right)$, using (2.5) and we have

$$
\begin{aligned}
g\left(A_{N Z} U_{\alpha}+A_{N T Z} F U_{\alpha}, V_{\alpha}\right)= & g\left(A_{N Z} U_{\alpha}, V_{\alpha}\right)+g\left(A_{N T Z} F U_{\alpha}, V_{\alpha}\right) \\
= & g\left(A_{N Z} V_{\alpha}, U_{\alpha}\right)+g\left(A_{N T Z} V_{\alpha}, F U_{\alpha}\right) \\
= & -g\left(\bar{\nabla}_{V_{\alpha}} N Z, U_{\alpha}\right)-g\left(\bar{\nabla}_{V_{\alpha}} N T Z, F U_{\alpha}\right) \\
= & -g\left(\bar{\nabla}_{V_{\alpha}} N Z, U_{\alpha}\right)-g\left(\bar{\nabla}_{V_{\alpha}} F T Z, F U_{\alpha}\right) \\
& +g\left(\bar{\nabla}_{V_{\alpha}} T^{2} Z, F U_{\alpha}\right) .
\end{aligned}
$$

Using (2.10), (2.11), (3.1) and (3.3), we arrive

$$
\begin{aligned}
g\left(A_{N Z} U_{\alpha}+A_{N T Z} F U_{\alpha}, V_{\alpha}\right)= & -g\left(\bar{\nabla}_{V_{\alpha}} F Z, U_{\alpha}\right)+g\left(\bar{\nabla}_{V_{\alpha}} T Z, U_{\alpha}\right)-g\left(\bar{\nabla}_{V_{\alpha}} T Z, U_{\alpha}\right) \\
& +\cos ^{2} \theta g\left(\bar{\nabla}_{V_{\alpha}} Z, F U_{\alpha}\right) \\
= & -g\left(\bar{\nabla}_{V_{\alpha}} F Z, U_{\alpha}\right)+\cos ^{2} \theta g\left(\bar{\nabla}_{V_{\alpha}} Z, F U_{\alpha}\right) .
\end{aligned}
$$

Using (2.3), (2.5), (2.9) (2.11), we find

$$
\begin{aligned}
g\left(A_{N Z} U_{\alpha}+A_{N T Z} F U_{\alpha}, V_{\alpha}\right) & =-g\left(\bar{\nabla}_{V_{\alpha}} Z, F U_{\alpha}\right)+\cos ^{2} \theta g\left(\nabla_{V_{\alpha}} Z, F U_{\alpha}\right) \\
& =-g\left(\nabla_{V_{\alpha}} Z, F U_{\alpha}\right)+\cos ^{2} \theta g\left(\nabla_{V_{\alpha}} Z, F U_{\alpha}\right) \\
& =-\sin ^{2} \theta g\left(\nabla_{V_{\alpha}} Z, F U_{\alpha}\right) \\
& =-\sin ^{2} \theta Z\left(\ln f_{\alpha}\right) g\left(V_{\alpha}, F U_{\alpha}\right) .
\end{aligned}
$$

So, we conclude that

$$
g\left(A_{N Z} U_{\alpha}+A_{N T Z} F U_{\alpha}, V_{\alpha}\right)=-\sin ^{2} \theta Z\left(\ln f_{\alpha}\right) g\left(F U_{\alpha}, V_{\alpha}\right) .
$$

Moreover, we have $X_{a}\left(\ln f_{\alpha}\right)=U_{\alpha}\left(\ln f_{\alpha}\right)=0$, since $f$ depends on only points of $M_{\theta}$. So, we conclude that $\mu=\ln f_{\alpha}$. Thus from (5.13) (5.15), we get (5.2).

Next, we prove (5.3) (5.9). We know $M$ is a multiply warped product generalized semi-invariant submanifold of a l.p.R. manifold $(\bar{M}, F, g)$. Then, for $Z, W \in \Gamma\left(\mathcal{D}^{\theta}\right)$, using (2.2), we get $\nabla_{Z} W=\nabla_{Z}^{\theta} W$ and for $X_{a} \in \Gamma\left(\mathcal{D}_{a}^{\perp}\right)$, we have

$$
g\left(\nabla_{Z} W, X_{a}\right)=\sec ^{2} \theta\left\{g\left(A_{F X_{a}} Z, T W\right)+g\left(A_{N T W} Z, X_{a}\right)\right\}=g\left(\nabla_{Z}^{\theta} W, X_{a}\right)=0
$$

from (3.8). Since $M_{\theta}$ is a proper slant submanifold, it follows that

$$
g\left(A_{F X_{a}} Z, T W\right)+g\left(A_{N T W} Z, X_{a}\right)=0 .
$$

Which is (5.3). For $U_{\alpha}, V_{\alpha} \in \Gamma\left(\mathcal{D}_{\alpha}^{T}\right)$ and $X_{a} \in \Gamma\left(\mathcal{D}_{a}^{\perp}\right)$, using (2.4), we get $g\left(\nabla_{U_{\alpha}} V_{\alpha}, X_{a}\right)=g\left(\nabla_{U_{\alpha}}^{T} V_{\alpha}-\right.$ $\left.g\left(U_{\alpha}, V_{\alpha}\right) \nabla\left(\ln f_{\alpha}\right), X_{a}\right)=0$. Then from (3.10) we find

$$
g\left(\nabla_{U_{\alpha}} V_{\alpha}, X_{a}\right)=g\left(A_{F X_{a}} U_{\alpha}, F V_{\alpha}\right)=0 .
$$

Therefore, we get (5.4). For $U_{\alpha} \in \Gamma\left(\mathcal{D}_{\alpha}^{T}\right)$ and $X_{a}, Y_{a} \in \Gamma\left(\mathcal{D}_{a}^{\perp}\right)$, using (2.4), we have $g\left(\nabla_{X_{a}} Y_{a}, U_{\alpha}\right)=g\left(\nabla^{\perp} X_{a} Y_{a}-\right.$ $\left.g\left(X_{a}, Y_{a}\right) \nabla\left(\ln \sigma_{a}\right), U_{\alpha}\right)=0$. Then from (3.11) we find,

$$
g\left(\nabla_{X_{a}} Y_{a}, U_{\alpha}\right)=-g\left(A_{F Y_{a}} X_{a}, F U_{\alpha}\right)=0 .
$$

Hence, we conclude that (5.5). For $X_{a} \in \Gamma\left(\mathcal{D}_{a}^{\perp}\right), Z \in \Gamma\left(\mathcal{D}^{\theta}\right)$ and $U_{\alpha} \in \Gamma\left(\mathcal{D}_{\alpha}^{T}\right)$, using (2.3), we write $g\left(\nabla_{Z} X_{a}, F U_{\alpha}\right)=g\left(Z\left(\ln \sigma_{a}\right) X_{a}, F U_{\alpha}\right)=Z\left(\ln \sigma_{a}\right) g\left(X_{a}, F U_{\alpha}\right)=0$. On the other hand, from (3.13) we find

$$
g\left(\nabla_{Z} X_{a}, F U_{\alpha}\right)=-g\left(A_{F X_{a}} Z, F U_{\alpha}\right)=0 .
$$

Thus, we get (5.6). For $X_{a} \in \Gamma\left(\mathcal{D}_{a}^{\perp}\right), Z \in \Gamma\left(\mathcal{D}^{\theta}\right)$ and $U_{\alpha} \in \Gamma\left(\mathcal{D}_{\alpha}^{T}\right)$, using (2.4), we have $g\left(\nabla_{U_{\alpha}} X_{a}, Z\right)=0$. Then, from (3.14) we find,

$$
g\left(\nabla_{U_{\alpha}} X_{a}, Z\right)=-\sec ^{2} \theta\left\{g\left(A_{F X_{a}} U_{\alpha}, T Z\right)+g\left(A_{N T Z} U_{\alpha}, X_{a}\right)\right\}=0 .
$$

It follows that (5.7).

For $U_{\alpha}, V_{\alpha} \in \Gamma\left(\mathcal{D}_{\alpha}^{T}\right), U_{\beta} \in \Gamma\left(\mathcal{D}_{\beta}^{T}\right)$ and $U_{\gamma} \in \Gamma\left(\mathcal{D}_{\gamma}^{T}\right)$ for $1 \leq \alpha, \beta, \gamma \leq k$ with $\alpha \neq \beta$ and $\alpha \neq \gamma$ then we have $g\left(\nabla_{U_{\beta}} U_{\gamma}, U_{\alpha}\right)=0$ from (2.4). Hence, we get (5.8). For $X_{a}, Y_{a} \in \Gamma\left(\mathcal{D}_{a}^{\perp}\right), X_{b} \in \Gamma\left(\mathcal{D}_{b}^{\perp}\right)$ and $X_{c} \in \Gamma\left(\mathcal{D}_{c}^{\perp}\right)$ for $1 \leq a, b, c \leq l$ with $a \neq b$ and $a \neq c$ then we have $g\left(\nabla_{X_{b}} X_{c}, X_{a}\right)=0$ from (2.4). Thus, we get (5.9). Since $M$ is a multiply warped product generalized semi-invariant submanifold then all distrubutions involve in the 
definition must be integrable. Thus (3.22) and (3.25) respectively hold.

Conversely, assume that $M$ is a $\left(\mathcal{D}^{\theta}, \mathcal{D}_{a}^{\perp}\right)$-mixed geodesic multiply warped product generalized semiinvariant submanifold of l.p.R manifold $(\bar{M}, F, g)$ such that (5.1) (5.9) and (3.22) (3.25) hold. From (5.3), we satisfy (3.19). On the other hand if we write $F U_{\alpha}$ instead of $U_{\alpha}$ and $W$ instead of $Z$ in (5.2), we find $A_{N W} F U_{\alpha}+A_{N T W} U_{\alpha}=-\sin ^{2} \theta W(\mu) U_{\alpha}$. If we take inner product of this equation with $Z \in \Gamma\left(\mathcal{D}^{\theta}\right)$, we get

$$
\begin{aligned}
g\left(A_{N W} F U_{\alpha}+A_{N T W} U_{\alpha}, Z\right) & =g\left(A_{N W} Z, F U_{\alpha}\right)+g\left(A_{N T W} Z, U_{\alpha}\right) \\
& =-\sin ^{2} \theta W(\mu) g\left(U_{\alpha}, Z\right)=0 .
\end{aligned}
$$

So, (3.18) holds. Thus, the slant distribution $\mathcal{D}^{\theta}$ is totally geodesic and as a result it is integrable. On the other hand, from (5.4), for all $U_{\alpha}, V_{\alpha} \in \Gamma\left(\mathcal{D}_{\alpha}^{T}\right)$ and $X_{a} \in \Gamma\left(\mathcal{D}_{a}^{\perp}\right)$, we write $g\left(A_{F X_{a}} U_{\alpha}, F V_{\alpha}\right)=0$. Thus, $g\left(A_{F X_{a}} U_{\alpha}, F V_{\alpha}\right)=g\left(A_{F X_{a}} U_{\alpha}, F V_{\alpha}\right)$. Which is (3.20). On the other hand, in (5.2), if we write $F U_{\alpha}$ instead of $U_{\alpha}$, we find $A_{N Z} F U_{\alpha}+A_{N T Z} U_{\alpha}=-\sin ^{2} \theta Z(\mu) U_{\alpha}$. If we take inner product of this equation with $V_{\alpha} \in \Gamma\left(\mathcal{D}_{\alpha}^{T}\right)$, we arrive at

$$
\begin{aligned}
g\left(A_{N Z} F U_{\alpha}+A_{N T Z} U_{\alpha}, V_{\alpha}\right) & =g\left(A_{N Z} F U_{\alpha}, V_{\alpha}\right)+g\left(A_{N T Z} U_{\alpha}, V_{\alpha}\right) \\
& =-\sin ^{2} \theta Z(\mu) g\left(U_{\alpha}, V_{\alpha}\right) .
\end{aligned}
$$

Here, if we interchange $U_{\alpha}$ and $V_{\alpha}$ in (5.16), we find

$$
\begin{aligned}
g\left(A_{N Z} F U_{\alpha}+A_{N T Z} U_{\alpha}, V_{\alpha}\right) & =g\left(A_{N Z} F U_{\alpha}, V_{\alpha}\right)+g\left(A_{N T Z} U_{\alpha}, V_{\alpha}\right) \\
& =-\sin ^{2} \theta Z(\mu) g\left(U_{\alpha}, V_{\alpha}\right) .
\end{aligned}
$$

From (5.16) and (5.17), we get

$$
g\left(A_{N Z} U_{\alpha}, F V_{\alpha}\right)+g\left(A_{N T Z} U_{\alpha}, V_{\alpha}\right)=g\left(A_{N Z} V_{\alpha}, F U_{\alpha}\right)+g\left(A_{N T Z} V_{\alpha}, U_{\alpha}\right) .
$$

This is (3.21). We have already (3.22). Thus, by Theorem 3.2, the invariant distribution $\mathcal{D}_{\alpha}^{T}, 1 \leq \alpha \leq k$ is integrable. On the other hand, for all $X_{a}, Y_{a} \in \Gamma\left(\mathcal{D}_{a}^{\perp}\right)$ and $U_{\alpha} \in \Gamma\left(\mathcal{D}_{\alpha}^{T}\right)$, we have $g\left(A_{F Y_{a}} X_{a}, F U_{\alpha}\right)=0$ from (5.5). It follows that

$$
g\left(A_{F Y_{a}} X_{a}, F U_{\alpha}\right)=g\left(A_{F X_{a}} Y_{a}, F U_{\alpha}\right)=0 .
$$

That is (3.23). Also, we get

$g\left(\nabla_{X_{a}} Y_{a}, Z\right)=-\sec ^{2} \theta\left\{g\left(h\left(Y_{a}, T Z\right), X_{a}\right)+g\left(A_{N T Z} X_{a}, Y_{a}\right)\right\}$ from (3.15). Since $M$ is $\left(\mathcal{D}^{\theta}, \mathcal{D}_{a}^{\perp}\right)$-mixed geodesic, it follows that $g\left(h\left(Y_{a}, T Z\right), F X_{a}\right)=0$. Then, we find

$$
g\left(\nabla_{X_{a}} Y_{a}, Z\right)=g\left(\nabla_{Y_{a}} X_{a}, Z\right) .
$$

Thus (3.24) follows. We have already (3.25). Thus by Theorem 3.3, the totally real distributions $\mathcal{D}_{a}^{\perp}, 1 \leq a \leq l$ is integrable. Let $M_{\theta}, M_{\alpha}^{T}$ and $M_{a}^{\perp}$ be the integral manifolds of $\mathcal{D}^{\theta}, \mathcal{D}_{\alpha}^{T}$ and $\mathcal{D}_{a}^{\perp}$ respectively. If we denote the second fundamental form of $M_{\alpha}^{T}$ in $M$ by $h_{\alpha}^{T}$, for $U_{\alpha}, V_{\alpha} \in \Gamma\left(\mathcal{D}_{\alpha}^{T}\right)$ and $X_{a} \in \Gamma\left(\mathcal{D}_{a}^{\perp}\right)$, using (2.5), (3.10) and (5.4), we have

$$
g\left(h_{\alpha}^{T}\left(U_{\alpha}, V_{\alpha}\right), X_{a}\right)=g\left(\nabla_{U_{\alpha}} V_{\alpha}, X_{a}\right)=g\left(A_{F X_{a}} U_{\alpha}, F V_{\alpha}\right)=0 .
$$

For any $U_{\alpha}, V_{\alpha} \in \Gamma\left(\mathcal{D}_{\alpha}^{T}\right)$ and $Z \in \Gamma\left(\mathcal{D}^{\theta}\right)$, using (2.5) and (3.9), we get

$$
g\left(h_{\alpha}^{T}\left(U_{\alpha}, V_{\alpha}\right), Z\right)=g\left(\nabla_{U_{\alpha}} V_{\alpha}, Z\right)=\csc ^{2} \theta g\left(A_{N T Z} U_{\alpha}, V_{\alpha}\right)+g\left(A_{N Z} U_{\alpha}, F V_{\alpha}\right) .
$$

At this equation, if we use (5.2), we have

$$
g\left(h_{\alpha}^{T}\left(U_{\alpha}, V_{\alpha}\right), Z\right)=\csc ^{2} \theta\left\{g\left(A_{N T Z} V_{\alpha}+A_{N Z} F V_{\alpha}, U_{\alpha}\right)\right\}=-Z(\mu) g\left(V_{\alpha}, U_{\alpha}\right) .
$$

After some calculation, we obtain

$$
g\left(h_{\alpha}^{T}\left(U_{\alpha}, V_{\alpha}\right), Z\right)=g\left(-g\left(U_{\alpha}, V_{\alpha}\right) \nabla \mu, Z\right)
$$

where $\nabla \mu$ is the gradient of $\mu$. Thus, from (5.18) and (5.19), we conclude that

$$
h_{\alpha}^{T}\left(U_{\alpha}, V_{\alpha}\right)=-g\left(U_{\alpha}, V_{\alpha}\right) \nabla \mu .
$$

This equation says that $M_{\alpha}^{T}$ is totally umbilic in $M$ with the mean curvature vector field $-\nabla \mu$. Now, we show that $-\nabla \mu$ is parallel. We have to satisfy $g\left(\nabla_{U_{\alpha}} \nabla \mu, E\right)=0$ for $U_{\alpha} \in \Gamma\left(\mathcal{D}_{\alpha}^{T}\right)$ and $E \in\left(\mathcal{D}_{\alpha}^{T}\right)^{\perp}=\mathcal{D}^{\theta} \oplus \mathcal{D}_{1}^{\perp} \oplus \ldots \oplus$ 
$\mathcal{D}_{l}^{\perp} \oplus \mathcal{D}_{1}^{T} \oplus \ldots \oplus \hat{\mathcal{D}}_{\alpha}^{T} \oplus \ldots \oplus \mathcal{D}_{k}^{T}$, where the symbol ${ }^{\wedge}$ indicate the term to be omitted. Here, we can put $E=$ $Z+\sum_{a=1}^{l} X_{a}+\sum_{\beta=1}^{k} U_{\beta}$, where $Z \in \Gamma\left(\mathcal{D}^{\theta}\right), X_{a} \in \Gamma\left(\mathcal{D}_{a}^{\perp}\right)$ and $U_{\alpha} \in \Gamma\left(\mathcal{D}_{\alpha}^{T}\right), V_{\beta} \in \Gamma\left(\mathcal{D}_{\beta}^{T}\right)$ with $\alpha \neq \beta$, then we can write that $g\left(\nabla_{U_{\alpha}} \nabla \mu, E\right)=g\left(\nabla_{U_{\alpha}} \nabla \mu, Z\right)+\sum_{a=1}^{l} g\left(\nabla_{U_{\alpha}} \nabla \mu, X_{a}\right)+\sum_{\beta=1}^{k} g\left(\nabla_{U_{\alpha}} \nabla \mu, U_{\beta}\right)$. By direct computations, we obtain

$$
\begin{aligned}
g\left(\nabla_{U_{\alpha}} \nabla \mu, E\right) & =\left\{U_{\alpha} g(\nabla \mu, E)-g\left(\nabla \mu, \nabla_{U_{\alpha}} E\right)\right\} \\
& =U_{\alpha}(E(\mu))-\left[U_{\alpha}, E\right](\mu)-g\left(\nabla \mu, \nabla_{E} U_{\alpha}\right) \\
& =\left[U_{\alpha}, E\right](\mu)+E\left(U_{\alpha}(\mu)\right)-\left[U_{\alpha}, E\right](\mu)-g\left(\nabla \mu, \nabla_{E} U_{\alpha}\right) \\
& =-g\left(\nabla \mu, \nabla_{E} U_{\alpha}\right) \\
& =-g\left(\nabla \mu, \nabla_{Z} U_{\alpha}\right)-\sum_{a=1}^{l} g\left(\nabla \mu, \nabla_{X_{a}} U_{\alpha}\right)-\sum_{\beta=1}^{k} g\left(\nabla \mu, \nabla_{U_{\beta}} U_{\alpha}\right),
\end{aligned}
$$

since $U_{\alpha}(\mu)=0$. Here, for any $W \in \Gamma\left(\mathcal{D}^{\theta}\right)$, we have $g\left(\nabla_{Z} U_{\alpha}, W\right)=-g\left(U_{\alpha}, \nabla_{Z} W\right)=0$, since $M_{\theta}$ is totally geodesic in $M$. Thus, $\nabla_{Z} U_{\alpha} \in \Gamma\left(\mathcal{D}_{\alpha}^{T}\right)$ or $\nabla_{Z} U_{\alpha} \in \Gamma\left(\mathcal{D}_{a}^{\perp}\right)$. In either case, we have $g\left(\nabla \mu, \nabla_{Z} U_{\alpha}\right)=0$. And then we get

$$
g\left(\nabla_{U_{\alpha}} \nabla \mu, Z\right)=0 .
$$

By the same way like (5.20) we get $\sum_{a=1}^{l} g\left(\nabla_{U_{\alpha}} \nabla \mu, X_{a}\right)=-\sum_{a=1}^{l} g\left(\nabla \mu, \nabla_{X_{a}} U_{\alpha}\right)$.

On the other hand, from (3.12), we have $g\left(\nabla_{X_{a}} U_{\alpha}, W\right)=-g\left(U_{\alpha}, \nabla_{X_{a}} W\right)=$ $-\csc ^{2} \theta\left\{g\left(A_{N T W} X_{a}, U_{\alpha}\right)+g\left(A_{N W} X_{a}, F U_{\alpha}\right)\right\}$. Here, using (5.2), we obtain

$$
g\left(\nabla_{X_{a}} U_{\alpha}, W\right)=g\left(W(\mu) U_{\alpha}, X_{a}\right)=0 .
$$

That is; $\nabla_{X_{a}} U_{\alpha} \in \Gamma\left(\mathcal{D}_{\alpha}^{T}\right)$ or $\nabla_{X_{a}} U_{\alpha} \in \Gamma\left(\mathcal{D}_{a}^{\perp}\right)$. In either case, we get $g\left(\nabla \mu, \nabla_{X_{a}} U_{\alpha}\right)=0$. And then we find

$$
\sum_{a=1}^{l} g\left(\nabla_{U_{\alpha}} \nabla \mu, X_{a}\right)=0 .
$$

Here using (2.4), directly we get $g\left(\nabla_{U_{\beta}} U_{\alpha}, W\right)=0$, directly we conclude that:

$$
\sum_{\beta=1}^{k} g\left(\nabla_{U_{\beta}} \nabla \mu, U_{\alpha}\right)=0 .
$$

And then from (5.20), (5.21) and (5.22) we find

$$
g\left(\nabla_{U_{\alpha}} \nabla \mu, E\right)=0 .
$$

Thus, $M_{\alpha}^{T}$ is spherical, since it is also totally umbilic. Consequently, $\mathcal{D}_{\alpha}^{T}$ is spherical, for $1 \leq \alpha \leq k$.

Next, we show that $\mathcal{D}_{a}^{\perp}$ is spherical. Let $h_{a}^{\perp}$ denote the second fundamental form of $M_{a}^{\perp}$ in $M$. Then for $X_{a}, Y_{a} \in \Gamma\left(\mathcal{D}_{a}^{\perp}\right)$ and $U_{\alpha} \in \Gamma\left(\mathcal{D}_{\alpha}^{T}\right)$, using (2.5), (3.11) and (5.5), we have

$$
g\left(h_{a}^{\perp}\left(X_{a}, Y_{a}\right), U_{\alpha}\right)=g\left(\nabla_{X_{a}} Y_{a}, U_{\alpha}\right)=-g\left(A_{F Y_{a}} X_{a}, F U_{\alpha}\right)=0 .
$$

On the other hand, for any $Z \in \Gamma\left(\mathcal{D}^{\theta}\right)$, using (3.15)

$$
g\left(h_{a}^{\perp}\left(X_{a}, Y_{a}\right), Z\right)=-\sec ^{2} \theta\left\{g\left(h\left(X_{a}, T Z\right), F Y_{a}\right)+g\left(A_{N T Z} X_{a}, Y_{a}\right)\right\} .
$$

Since $M$ is $\left(\mathcal{D}^{\theta}, \mathcal{D}_{a}^{\perp}\right)$-mixed geodesic, $g\left(h_{a}\left(X_{a}, T Z\right), F Y_{a}\right)=0$. So, we have

$$
g\left(h_{a}^{\perp}\left(X_{a}, Y_{a}\right), Z\right)=-g\left(A_{N T Z} X_{a}, Y_{a}\right) .
$$

Using (5.1), we obtain

$$
g\left(h_{a}^{\perp}\left(X_{a}, Y_{a}\right), Z\right)=-Z(\lambda) g\left(X_{a}, Y_{a}\right) .
$$

After some calculation, we get

$$
g\left(h_{a}^{\perp}\left(X_{a}, Y_{a}\right), Z\right)=-g\left(g\left(X_{a}, Y_{a}\right) \nabla \lambda, Z\right),
$$

where $\nabla \lambda$ is the gradient of $\lambda$. Thus, from (5.23) and (5.24), we deduce that

$$
h_{a}^{\perp}\left(X_{a}, Y_{a}\right)=-g\left(X_{a}, Y_{a}\right) \nabla \lambda .
$$

It means that $M_{a}^{\perp}$ is totally umbilic in $M$ with the mean curvature vector field $-\nabla \lambda$. What's left is to show that $-\nabla \lambda$ is parallel. We have to satisfy $g\left(\nabla_{X_{a}} \nabla \lambda, E\right)=0$ for $X_{a}, Y_{a} \in \Gamma\left(\mathcal{D}_{a}^{\perp}\right)$ and 
$E \in\left(\mathcal{D}_{a}^{\perp}\right)^{\perp}=\mathcal{D}^{\theta} \oplus \mathcal{D}_{1}^{T} \oplus \ldots \oplus \mathcal{D}_{k}^{T} \oplus \mathcal{D}_{1}^{\perp} \oplus \ldots \hat{\mathcal{D}}_{a}^{\perp} \oplus \ldots \oplus \mathcal{D}_{l}^{\perp}$. The proof is similar parallelity of $-\nabla \mu$. So we omit it. $-\nabla \lambda$ is parallel. So, $M_{a}^{\perp}$ is spherical, since it is also totally umbilic. Consequently, $\mathcal{D}_{a}^{\perp}$ is spherical, for $1 \leq a \leq l$.

Lastly, we prove that $\left(\mathcal{D}_{\alpha}^{T}\right)^{\perp}=\mathcal{D}^{\theta} \oplus \mathcal{D}_{1}^{\perp} \oplus \ldots \oplus \mathcal{D}_{l}^{\perp} \oplus \mathcal{D}_{1}^{T} \oplus \ldots \oplus \hat{\mathcal{D}}_{\alpha}^{T} \oplus \ldots \oplus \mathcal{D}_{k}^{T}$ and $\left(\mathcal{D}_{a}^{\perp}\right)^{\perp}=\mathcal{D}^{\theta} \oplus \mathcal{D}_{1}^{T} \oplus$ $\ldots \oplus \mathcal{D}_{k}^{T} \oplus \mathcal{D}_{1}^{\perp} \oplus \cdots \hat{\mathcal{D}}_{a}^{\perp} \oplus \ldots \oplus \mathcal{D}_{l}^{\perp}$ are autoparallel, where the symbol' indicate the term to be omitted. In fact, $\mathcal{D}^{\theta} \oplus \mathcal{D}_{1}^{\perp} \ldots \oplus \mathcal{D}_{l}^{\perp} \oplus \mathcal{D}_{1}^{T} \oplus \ldots \oplus \hat{\mathcal{D}}_{\alpha}^{T} \oplus \ldots \oplus \mathcal{D}_{k}^{T}$ is autoparallel if and only if for all types of covariant derivatives $\nabla_{Z} W, \nabla_{Z} X_{a}, \nabla_{X_{a}} Z, \nabla_{X_{a}} Y_{a}, \nabla_{U_{\beta}} Z, \nabla_{U_{\beta}} X_{a}, \nabla_{U_{\beta}} U_{\gamma}$ are again in $\Gamma\left(\mathcal{D}^{\theta} \oplus \mathcal{D}_{1}^{\perp} \oplus \ldots \oplus \mathcal{D}_{l}^{\perp} \oplus \mathcal{D}_{1}^{T} \oplus \ldots \oplus \hat{\mathcal{D}}_{\alpha}^{T} \oplus \ldots \oplus\right.$ $\mathcal{D}_{k}^{T}$ ) for $Z, W \in \Gamma\left(\mathcal{D}^{\theta}\right)$ and $X_{a}, Y_{a} \in \Gamma\left(\mathcal{D}_{a}^{\perp}\right)$. It means that all seven inner products $g\left(\nabla_{Z} W, U_{\alpha}\right), g\left(\nabla_{Z} X_{a}, U_{\alpha}\right)$, $g\left(\nabla_{X_{a}} Z, U_{\alpha}\right), g\left(\nabla_{X_{a}} Y_{a}, U_{\alpha}\right), g\left(\nabla_{U_{\beta}} Z, U_{\alpha}\right), g\left(\nabla_{U_{\beta}} X_{a}, U_{\alpha}\right), g\left(\nabla_{U_{\beta}} U_{\gamma}, U_{\alpha}\right)$, vanish, where $U_{\alpha} \in \Gamma\left(\mathcal{D}_{\alpha}^{T}\right), U_{\beta} \in \Gamma\left(\mathcal{D}_{\beta}^{T}\right)$ with $\alpha \neq \beta$ and $\alpha \neq \gamma, X_{a}, Y_{a} \in \Gamma\left(\mathcal{D}_{\alpha}^{T}\right)$ and $Z \in \Gamma\left(\mathcal{D}_{\theta}^{T}\right)$. Using (3.7) and (5.2), we get

$$
\begin{aligned}
g\left(\nabla_{Z} W, U_{\alpha}\right) & =-\csc ^{2} \theta\left\{g\left(A_{N T W} Z, U_{\alpha}\right)+g\left(A_{N W} Z, F U_{\alpha}\right)\right\} \\
& =-\csc ^{2} \theta g\left(A_{N T W} U_{\alpha}+A_{N W} F U_{\alpha}, Z\right) \\
& =W(\mu) g\left(U_{\alpha}, Z\right)=0 .
\end{aligned}
$$

Using (3.13) and (5.6), we find

$$
g\left(\nabla_{Z} X_{a}, U_{\alpha}\right)=-g\left(A_{F X_{a}} Z, F U_{\alpha}\right)=0 .
$$

By (3.12) and (5.2), we get

$$
g\left(\nabla_{X_{a}} Z, U_{\alpha}\right)=-\csc ^{2} \theta\left\{g\left(A_{N T Z} X_{a}, U_{\alpha}\right)+g\left(A_{N Z} X_{a}, F U_{\alpha}\right)\right\}=0 .
$$

By (3.11) and (5.5), we find

$$
g\left(\nabla_{X_{a}} Y_{a}, U_{\alpha}\right)=-g\left(A_{F Y_{a}} X_{a}, F U_{\alpha}\right)=0 .
$$

By (3.10) and (5.4), we find

$$
g\left(\nabla_{U_{\beta}} X_{a}, U_{\alpha}\right)=-g\left(X_{a}, \nabla_{U_{\beta}} U_{\alpha}\right)=-g\left(A_{F X_{a}} U_{\beta}, F U_{\alpha}\right)=0 .
$$

From (2.4), we find

$$
g\left(\nabla_{U_{\beta}} U_{\gamma}, U_{\alpha}\right)=0 .
$$

Thus, $\quad \mathcal{D}^{\theta} \oplus \mathcal{D}_{1}^{\perp} \oplus \ldots \oplus \mathcal{D}_{l}^{\perp} \oplus \mathcal{D}_{1}^{T} \oplus \ldots \oplus \hat{\mathcal{D}}_{\alpha}^{T} \oplus \ldots \oplus \mathcal{D}_{k}^{T} \quad$ is autoparallel. On the other hand, $\mathcal{D}^{\theta} \oplus$ $\mathcal{D}_{1}^{T} \oplus \cdots \oplus \mathcal{D}_{k}^{T} \oplus \mathcal{D}_{1}^{\perp} \oplus \ldots \hat{\mathcal{D}}_{a}^{\perp} \oplus \ldots \oplus \mathcal{D}_{l}^{\perp}$ is autoparallel if and only if all seven inner products $g\left(\nabla_{Z} W, X_{a}\right), g\left(\nabla_{Z} U_{\alpha}, X_{a}\right), g\left(\nabla_{U} Z, X_{a}\right), g\left(\nabla_{U_{\alpha}} V_{\alpha}, X_{a}\right), g\left(\nabla_{U_{\alpha}} V_{\beta}, X_{a}\right), g\left(\nabla_{Y_{b}} Z_{b}, X_{a}\right), g\left(\nabla_{Y_{b}} Z_{c}, X_{a}\right)$ vanish, where $Z, W \in \Gamma\left(\mathcal{D}^{\theta}\right), U_{\alpha}, V_{\alpha} \in \Gamma\left(\mathcal{D}_{\alpha}^{T}\right), V_{\beta} \in \Gamma\left(\mathcal{D}_{\beta}^{T}\right)$ for $\alpha \neq \beta, X_{a} \in \Gamma\left(\mathcal{D}_{a}^{\perp}\right), Y_{b} \in \Gamma\left(\mathcal{D}_{b}^{\perp}\right)$ for $a \neq b$ and $Z_{b}, Z_{c} \in \Gamma\left(\mathcal{D}_{\theta}^{T}\right)$ with $b \neq c$. Firstly, we have already $g\left(\nabla_{Z} U_{\alpha}, X_{a}\right)=0$ from above. Using (3.8) and (5.3), we get

$$
g\left(\nabla_{Z} W, X_{a}\right)=\sec ^{2} \theta\left\{g\left(A_{F X_{a}} Z, T W\right)+g\left(A_{N T W} Z, X_{a}\right)\right\}=0 .
$$

Using (3.10) and (5.4), we find

Directly we conclude that

$$
g\left(\nabla_{U_{\alpha}} V_{\alpha}, X_{a}\right)=g\left(A_{F X_{a}} U_{\alpha}, F V_{\alpha}\right)=0
$$

$$
g\left(\nabla_{U_{\alpha}} V_{\beta}, X_{a}\right)=g\left(A_{F X_{a}} U_{\alpha}, F V_{\beta}\right)=0
$$

Using (3.10) and (5.4), we find

$$
g\left(\nabla_{Y_{b}} Z_{b}, X_{a}\right)=0, \quad g\left(\nabla_{Y_{b}} Z_{c}, X_{a}\right)=0 .
$$

And then, by (3.14) and (5.7), we get

$$
g\left(\nabla_{U_{\alpha}} Z, X_{a}\right)=-g\left(\nabla_{U_{\alpha}} X_{a}, Z\right)=\sec ^{2} \theta\left\{g\left(A_{F X_{a}} U_{\alpha}, T Z\right)+g\left(A_{N T Z} U_{\alpha}, X_{a}\right)\right\}=0 .
$$

So, $\mathcal{D}^{\theta} \oplus \mathcal{D}_{1}^{T} \oplus \ldots \oplus \mathcal{D}_{k}^{T} \oplus \mathcal{D}_{1}^{\perp} \oplus \ldots \hat{\mathcal{D}}_{a}^{\perp} \oplus \ldots \oplus \mathcal{D}_{l}^{\perp}$ is autoparallel. Thus by Remark $5.1, M$ is locally multiply warped product generalized semi-invaraint submanifold of the form $M^{\theta} \times_{f_{1}} M_{1}^{T} \times \ldots \times{ }_{f_{k}} M_{k}^{T} \times_{\sigma_{1}} M_{1}^{\perp} \times$ $\ldots \times_{\sigma_{l}} M_{l}^{\perp}$.

Next, we investigate the behavior of the second fundamental form $h$ of a non-trivial multiply warped product generalized semi-invariant submanifold of a l.p.R. manifold $(\bar{M}, F, g)$ in the form $M^{\theta} \times_{f_{1}} M_{1}^{T} \times \cdots \times f_{f_{k}} M_{k}^{T} \times_{\sigma_{1}}$ $M_{1}^{\perp} \times \cdots \times \times_{\sigma_{l}} M_{l}^{\perp}$. 
Lemma 5.1. Let $M$ be a multiply warped product generalized semi-invariant submanifold in the form $M^{\theta} \times{ }_{f_{1}} M_{1}^{T} \times$ $\ldots \times_{f_{k}} M_{k}^{T} \times_{\sigma_{1}} M_{1}^{\perp} \times \ldots \times_{\sigma_{l}} M_{l}^{\perp}$ of a l.p.R. manifold $(\bar{M}, F, g)$. Then for the second fundamental form $h$ of $M$ in $(\bar{M}, F, g)$, we have

$$
\begin{aligned}
& g\left(h\left(U_{\alpha}, V_{\alpha}\right), N W\right)=-W\left(\ln f_{\alpha}\right) g\left(U_{\alpha}, F V_{\alpha}\right)+T W\left(\ln f_{\alpha}\right) g\left(U_{\alpha}, V_{\alpha}\right), \\
& g\left(h\left(Z, U_{\alpha}\right), N W\right)=0, \\
& g\left(h\left(X_{a}, U_{\alpha}\right), N W\right)=0, \\
& g\left(h\left(Z, U_{\alpha}\right), F X_{a}\right)=0, \\
& g\left(h\left(X_{a}, U_{\alpha}\right), F Y_{a}\right)=0, \\
& g\left(h\left(U_{\alpha}, V_{\alpha}\right), F X_{a}\right)=0, \\
& g\left(h\left(X_{a}, Y_{a}\right), N W\right)=-g\left(h\left(X_{a}, W\right), F Y_{a}\right)+T W\left(\ln \sigma_{a}\right) g\left(X_{a}, Y_{a}\right),
\end{aligned}
$$

where $Z, W \in \Gamma\left(\mathcal{D}^{\theta}\right), X_{a}, Y_{a} \in \Gamma\left(\mathcal{D}_{a}^{\perp}\right), Y_{b} \in \Gamma\left(\mathcal{D}_{b}^{\perp}\right)$ for $1 \leq a, b, c \leq l$ and $U_{\alpha}, V_{\alpha} \in \Gamma\left(\mathcal{D}_{\alpha}^{T}\right), V_{\beta} \in \Gamma\left(\mathcal{D}_{\beta}^{T}\right)$ for $1 \leq \alpha, \beta \leq$ $k$.

Proof. The proofs are very similar to the proofs of Lemmas 1, 2 and 3 of [22] and Lemma 5.3 of [13].

Lemma 5.2. Let $M$ be a multiply warped product generalized semi-invariant submanifold in the form $M^{\theta} \times{ }_{f_{1}} M_{1}^{T} \times$ $\ldots \times_{f_{k}} M_{k}^{T} \times_{\sigma_{1}} M_{1}^{\perp} \times \ldots \times_{\sigma_{l}} M_{l}^{\perp}$ of a l.p.R. manifold $(\bar{M}, F, g)$. Then for the second fundamental form $h$ of $M$ in $(\bar{M}, F, g)$, we have

$$
\begin{aligned}
& g\left(h\left(U_{\alpha}, V_{\beta}\right), N W\right)=0, \\
& g\left(h\left(U_{\alpha}, V_{\beta}\right), F X_{a}\right)=0, \\
& g\left(h\left(X_{a}, Y_{b}\right), N W\right)=0,
\end{aligned}
$$

where $Z, W \in \Gamma\left(\mathcal{D}^{\theta}\right), X_{a}, Y_{a} \in \Gamma\left(\mathcal{D}_{a}^{\perp}\right), Y_{b} \in \Gamma\left(\mathcal{D}_{b}^{\perp}\right)$ for $1 \leq a, b, c \leq l$, with $a \neq b$ and $U_{\alpha}, V_{\alpha} \in \Gamma\left(\mathcal{D}_{\alpha}^{T}\right), V_{\beta} \in \Gamma\left(\mathcal{D}_{\beta}^{T}\right)$ for $1 \leq \alpha, \beta \leq k$ with $\alpha \neq \beta$.

Proof. For $U_{\alpha} \in \Gamma\left(\mathcal{D}_{\alpha}^{T}\right), V_{\beta} \in \Gamma\left(\mathcal{D}_{\beta}^{T}\right)$ with $\alpha \neq \beta$ and $W \in \Gamma\left(\mathcal{D}^{\theta}\right)$ using (2.3), (2.5), (2.9) (2.11) and (3.1), we have

$$
\begin{aligned}
g\left(h\left(U_{\alpha}, V_{\beta}\right), N W\right) & =g\left(\bar{\nabla}_{U_{\alpha}} V_{\beta}, N W\right)=-g\left(V_{\beta}, \bar{\nabla}_{U_{\alpha}} N W\right) \\
& =-g\left(V_{\beta}, \nabla_{U_{\alpha}} F W\right)+g\left(V_{\beta}, \bar{\nabla}_{U_{\alpha}} T W\right) \\
& =-g\left(F V_{\beta}, \bar{\nabla}_{U_{\alpha}} W\right)+g\left(V_{\beta}, \nabla_{U_{\alpha}} T W\right) \\
& =-g\left(F V_{\beta}, \nabla_{U_{\alpha}} W\right)+g\left(V_{\beta}, \nabla_{U_{\alpha}} T W\right) \\
& =-W\left(\ln f_{\alpha}\right) g\left(F V_{\beta}, U_{\alpha}\right)+T W\left(\ln f_{\alpha}\right) g\left(U_{\alpha}, V_{\beta}\right) .
\end{aligned}
$$

Here $F V_{\beta} \in \Gamma\left(\mathcal{D}_{\beta}^{T}\right)$, then we have $g\left(U_{\alpha}, V_{\beta}\right)=0$ and $g\left(U_{\alpha}, F V_{\beta}\right)=0$ therefore $g\left(h\left(U_{\alpha}, V_{\beta}\right), N W\right)=0$. Hence we obtain (5.32). By using (2.5), (2.9) (2.11), (3.1) and (2.4) we get

$$
\begin{aligned}
g\left(h\left(U_{\alpha}, V_{\beta}\right), F X_{a}\right) & =g\left(\bar{\nabla}_{U_{\alpha}} V_{\beta}, F X_{a}\right)=-g\left(V_{\beta}, \bar{\nabla}_{U_{\alpha}} F X_{a}\right) \\
& =-g\left(F V_{\beta}, \bar{\nabla}_{U_{\alpha}} X_{a}\right)=-g\left(F V_{\beta}, \nabla_{U_{\alpha}} X_{a}\right)=0,
\end{aligned}
$$

for $U_{\alpha} \in \Gamma\left(\mathcal{D}_{\alpha}^{T}\right), V_{\beta} \in \Gamma\left(\mathcal{D}_{\beta}^{T}\right)$ with $\alpha \neq \beta$ and $X_{a} \in \Gamma\left(\mathcal{D}_{a}^{\perp}\right)$. Hence, we get (5.33). For $X_{a} \in \Gamma\left(\mathcal{D}_{a}^{\perp}\right), Y_{b} \in \Gamma\left(\mathcal{D}_{b}^{\perp}\right)$ with $a \neq b$ and $W \in \Gamma\left(\mathcal{D}^{\theta}\right)$ using (2.3), (2.5), (2.9) (2.11) and (3.1), we have

$$
\begin{aligned}
g\left(h\left(X_{a}, Y_{b}\right), N W\right) & =g\left(\bar{\nabla}_{X_{a}} Y_{b}, N W\right)=-g\left(Y_{b}, \bar{\nabla}_{X_{a}} N W\right) \\
& =-g\left(Y_{b}, \nabla_{X_{a}} F W\right)+g\left(Y_{b}, \bar{\nabla}_{X_{a}} T W\right) \\
& =-g\left(F Y_{b}, \bar{\nabla}_{X_{a}} W\right)+g\left(Y_{b}, \nabla_{X_{a}} T W\right) \\
& =g\left(\nabla_{X_{a}} F Y_{Y_{b}}, W\right)+g\left(Y_{b}, \nabla_{X_{a}} T W\right) \\
& =-g\left(A_{F Y_{b}} X_{a}, W\right)+T W\left(\ln \sigma_{a}\right) g\left(X_{a}, Y_{b}\right) \\
& =-g\left(h\left(X_{a}, W\right), F Y_{b}\right)+T W\left(\ln \sigma_{a}\right) g\left(X_{a}, Y_{b}\right) .
\end{aligned}
$$

Using the fact that $g\left(X_{a}, Y_{b}\right)=0$ and $g\left(h\left(X_{a}, W\right), F Y_{b}\right)=0$, consequently we get $g\left(h\left(X_{a}, Y_{b}\right), N W\right)=0$. Thus, we get (5.34). 
Lemmas 5.1 and 5.2 show us partially the behavior of the second fundamental form $h$ of the multiply warped product generalized semi-invariant submanifold has the form $M^{\theta} \times_{f_{1}} M_{1}^{T} \times \cdots \times \times_{f_{k}} M_{k}^{T} \times_{\sigma_{1}} M_{1}^{\perp} \times \cdots \times_{\sigma_{l}} M_{l}^{\perp}$ in the normal subbundles $N\left(\mathcal{D}^{\theta}\right)$ and $F\left(\mathcal{D}_{1}^{\perp}\right) \oplus \ldots \oplus F\left(\mathcal{D}_{l}^{\perp}\right.$. By using (5.26) (5.29) and (5.32) (5.33), we immediately have the following result.

Corollary 5.1. Let $M$ be a multiply warped-product generalized semi-invariant submanifold in the form $M^{\theta} \times_{f_{1}} M_{1}^{T} \times$ $\ldots \times_{f_{k}} M_{k}^{T} \times_{\sigma_{1}} M_{1}^{\perp} \times \ldots \times_{\sigma_{l}} M_{l}^{\perp}$ of a l.p.R. manifold $(\bar{M}, F, g)$ such that the invariant normal subbundle $\overline{\mathcal{D}}^{T}=\{0\}$. Then $M$ is $\left(\mathcal{D}_{\alpha}^{T}, \mathcal{D}_{a}^{\perp}\right),\left(\mathcal{D}_{\alpha}^{T}, \mathcal{D}^{\theta}\right)$ and $\left(\mathcal{D}_{\alpha}^{T}, \mathcal{D}_{\beta}^{T}\right)$-mixed geodesic, for $1 \leq \alpha, \beta \leq k$ with $\alpha \neq \beta$ and $1 \leq a, b \leq l$.

Lastly, we give another main result of this section.

Theorem 5.2. Let $M$ be a multiply warped product $\left(\mathcal{D}^{\theta}, \mathcal{D}_{a}^{\perp}\right)$-mixed geodesic generalized semi-invariant submanifold in the form $M^{\theta} \times_{f_{1}} M_{1}^{T} \times \ldots \times_{f_{k}} M_{k}^{T} \times_{\sigma_{1}} M_{1}^{\perp} \times \ldots \times_{\sigma_{l}} M_{l}^{\perp}$ of a l.p.R. manifold $(\bar{M}, F, g)$ such that its invariant normal subbundle $\overline{\mathcal{D}}^{T}=\{0\}$. Then $M$ is a locally multiply direct product in the form $M^{\theta} \times M_{l}^{T} \times \ldots \times M_{k}^{T} \times M_{1}^{\perp} \times \ldots \times M_{l}^{\perp}$ if and only if $g\left(h\left(\mathcal{D}_{a}^{\perp}, \mathcal{D}_{a}^{\perp}\right), N \mathcal{D}^{\theta}\right)=0$ and $M$ is $\mathcal{D}_{\alpha}^{T}$-geodesic, where $1 \leq \alpha \leq k$ and $1 \leq a \leq l$.

Proof. Let $M$ be a multiply warped product $\left(\mathcal{D}^{\theta}, \mathcal{D}_{a}^{\perp}\right)$-mixed geodesic generalized semi-invariant submanifold in the form $M^{\theta} \times_{f_{1}} M_{1}^{T} \times \ldots \times_{f_{k}} M_{k}^{T} \times_{\sigma_{1}} M_{1}^{\perp} \times \ldots \times_{\sigma_{l}} M_{l}^{\perp}$ of a 1.p.R. manifold $(\bar{M}, F, g)$ such that its invariant normal subbundle $\overline{\mathcal{D}}^{T}=\{0\}$. If $M$ is a locally multiply direct product in the form $M^{\theta} \times M_{l}^{T} \times \ldots \times M_{k}^{T} \times M_{1}^{\perp} \times \ldots \times M_{l}^{\perp}$, for $1 \leq \alpha \leq k$ and $1 \leq a \leq l$, the warping functions $f_{\alpha}$ and $\sigma_{a}$ are constants. By (5.31) and the fact that $M$ is $\left(\mathcal{D}^{\theta}, \mathcal{D}_{a}^{\perp}\right)$-mixed geodesic for $1 \leq a \leq l$, we have $g\left(h\left(X_{a}, Y_{a}\right), N W\right)=0$ for $X_{a}, Y_{a} \in \Gamma\left(\mathcal{D}_{a}^{\perp}\right)$ and $W \in \Gamma\left(\mathcal{D}^{\theta}\right)$. Which means that $g\left(h\left(\mathcal{D}_{a}^{\perp}, \mathcal{D}_{a}^{\perp}\right), N \mathcal{D}^{\theta}\right)=0$. On the other hand, using (5.25). we have $g\left(h\left(U_{\alpha}, V_{\alpha}\right), N W\right)=0$ for $U_{\alpha}, V_{\alpha} \in \Gamma\left(\mathcal{D}_{\alpha}^{T}\right)$ and $W \in \Gamma\left(\mathcal{D}^{\theta}\right)$, since $W\left(\ln f_{\alpha}\right)=T W\left(\ln f_{\alpha}\right)=0$ for $1 \leq \alpha \leq k$. Using this fact and (5.30), it follows that $h\left(U_{\alpha}, V_{\alpha}\right)=0$ for $U_{\alpha}, V_{\alpha} \in \Gamma\left(\mathcal{D}_{\alpha}^{T}\right)$. Which says us $M$ is $\mathcal{D}_{\alpha}^{\bar{T}}$-geodesic for $1 \leq \alpha \leq k$.

Conversely, let $g\left(h\left(\mathcal{D}_{a}^{\perp}, \mathcal{D}_{a}^{\perp}\right), N \mathcal{D}^{\theta}\right)=0$ and $M$ is $\mathcal{D}_{\alpha}^{T}$ - geodesic for $1 \leq \alpha \leq k$ and $1 \leq a \leq l$. Then, we have $T W\left(\ln \sigma_{a}\right)=0$ from (5.31), where $W \in \Gamma\left(\mathcal{D}^{\theta}\right)$. Hence, it follows that $\sigma_{a}$ is a constant for $1 \leq a \leq l$. On the other hand, for any $U_{\alpha}, V_{\alpha} \in \Gamma\left(\mathcal{D}_{\alpha}^{T}\right)$ and $W \in \Gamma\left(\mathcal{D}^{\theta}\right)$, we have

$$
W\left(\ln f_{\alpha}\right) g\left(U_{\alpha}, F V_{\alpha}\right)=T W\left(\ln f_{\alpha}\right) g\left(U_{\alpha}, V_{\alpha}\right)
$$

from (5.25). If we take $W=T W$ in (5.35) and using (3.3), we obtain

$$
T W\left(\ln f_{\alpha}\right) g\left(U_{\alpha}, F V_{\alpha}\right)=\cos ^{2} \theta W\left(\ln f_{\alpha}\right) g\left(U_{\alpha}, V_{\alpha}\right) .
$$

By replacing $V_{\alpha}$ by $F V_{\alpha}$ in (5.36), then (5.36) becomes

$$
T W\left(\ln f_{\alpha}\right) g\left(U_{\alpha}, V_{\alpha}\right)=\cos ^{2} \theta W\left(\ln f_{\alpha}\right) g\left(U_{\alpha}, F V_{\alpha}\right) .
$$

From (5.35) and (5.37), we get

$$
\cos ^{2} \theta W\left(\ln f_{\alpha}\right) g\left(U_{\alpha}, F V_{\alpha}\right)=0,
$$

for any $U_{\alpha}, V_{\alpha} \in \Gamma\left(\mathcal{D}_{\alpha}^{T}\right), 1 \leq \alpha \leq k$. Since $M$ is proper, $\cos \theta \neq 0$, we can deduce that $W\left(\ln f_{\alpha}\right)=0$ from (5.38). Namely, we find each $f_{\alpha}$ as a constant. Thus, $M$ must be a locally multiply direct product in the form $M^{\theta} \times M_{1}^{T} \times \ldots \times M_{k}^{T} \times M_{1}^{\perp} \times \ldots \times M_{l}^{\perp}$.

\section{An inequality for multiply warped product generalized semi-invariant submanifolds}

In this section, we shall establish an inequality for the squared norm of the second fundamental form in terms of the warping functions for multiply warped product generalized semi-invariant submanifold in the form $M^{\theta} \times_{f_{1}} M_{1}^{T} \times \ldots \times_{f_{k}} M_{k}^{T} \times_{\sigma_{1}} M_{1}^{\perp} \times \ldots \times_{\sigma_{l}} M_{l}^{\perp}$.

Remark 6.1. Let $M=M^{\theta} \times_{f_{1}} M_{l}^{T} \times \ldots \times_{f_{k}} M_{k}^{T} \times_{\sigma_{1}} M_{1}^{\perp} \times \ldots \times_{\sigma_{l}} M_{l}^{\perp}$ be a multiply warped product generalized semi-invariant submanifold of a l.p.R. manifold $(\bar{M}, F, g)$ and let $d=\operatorname{dim} M^{\theta}, m_{\alpha}=\operatorname{dim} M_{\alpha}^{T}$ and $n_{a}=\operatorname{dim} M_{a}^{\perp}$. We choose orthonormal basis of $M^{\theta}, M_{\alpha}^{T}$ and $M_{a}^{\perp}$, respectively as

$\left\{e_{1}^{*}=\sec \theta T e_{1}^{*}, \ldots, e_{d}^{*}=\sec \theta T e_{d}^{*}\right\}, \quad\left\{e_{1}^{\alpha}=F e_{1}^{\alpha}, \ldots, e_{z_{\alpha}}^{\alpha}=F e_{z_{\alpha}}^{\alpha}, e_{z_{\alpha+1}}^{\alpha}=-F e_{z_{\alpha+1}}^{\alpha} \ldots, e_{m_{\alpha}}^{\alpha}=-F e_{m_{\alpha}}^{\alpha}\right\} \quad$ and $\left\{w_{1}^{a}, \ldots, w_{n_{a}}^{a}\right\}$. Then the orthonormal basis of $N \mathcal{D}^{\theta}$ and $F \mathcal{D}_{a}^{\perp}$, respectively are $\left\{\overline{e_{1}}=\csc \theta N e_{1}^{*}, \ldots, \overline{e_{d}}=\csc \theta N e_{d}^{*}\right\}$ and $\left\{F w_{1}^{a}, \ldots, F w_{n_{a}}^{a}\right\}$, where $1 \leq \alpha \leq k$ and $1 \leq a \leq l$. 
Theorem 6.1. Let $M=M^{\theta} \times_{f_{1}} M_{1}^{T} \times \ldots \times_{f_{k}} M_{k}^{T} \times_{\sigma_{1}} M_{1}^{\perp} \times \ldots \times_{\sigma_{l}} M_{l}^{\perp}$ be a multiply warped product $\left(\mathcal{D}^{\theta}, \mathcal{D}_{a}^{\perp}\right)$ mixed geodesic generalized semi-invariant submanifold of a l.p.R. manifold $(\bar{M}, F, g)$ such that its invariant normal subbundle $\overline{\mathcal{D}}^{T}=\{0\}$. Then the squared norm of the second fundamental form of $M$ satisfies

$$
\begin{aligned}
\|h\|^{2} \geq & \cot ^{2} \theta \sum_{a=1}^{l} n_{a}\left\|\nabla\left(\ln \sigma_{a}\right)\right\|^{2} \\
& +\csc ^{2} \theta \sum_{\alpha=1}^{k}\left\|\nabla\left(\ln f_{\alpha}\right)\right\|^{2}\left((1+\cos \theta)^{2} m_{\alpha}-4 \cos \theta z_{\alpha}\right),
\end{aligned}
$$

where $n_{a}=\operatorname{dim} M_{a}^{\perp}, m_{\alpha}=\operatorname{dim} M_{\alpha}^{T}$ and $z_{\alpha}$ as in Remark 6.1. Moreover, the equality sign in (6.1) holds, identically if and only if $M^{\theta}$ is also totally geodesic in $\bar{M}$.

Proof. Let $M=M^{\theta} \times_{f_{1}} M_{1}^{T} \times \ldots \times_{f_{k}} M_{k}^{T} \times_{\sigma_{1}} M_{1}^{\perp} \times \ldots \times_{\sigma_{l}} M_{l}^{\perp}$ be a multiply warped product $\left(\mathcal{D}^{\theta}, \mathcal{D}_{1 \leq a \leq l}^{\perp}\right)$ mixed geodesic generalized semi-invariant submanifold of a l.p.R. manifold $(\bar{M}, F, g)$ such that its invariant normal subbundle $\overline{\mathcal{D}}^{T}=\{0\}$. From (2.8), we derive

$$
\begin{aligned}
\|h\|^{2}= & \left\|h\left(\mathcal{D}^{\theta}, \mathcal{D}^{\theta}\right)\right\|^{2}+\sum_{\alpha=1}^{k}\left\|h\left(\mathcal{D}_{\alpha}^{T}, \mathcal{D}_{\alpha}^{T}\right)\right\|^{2} \\
& +\sum_{a=1}^{l}\left\|h\left(\mathcal{D}_{a}^{\perp}, \mathcal{D}_{a}^{\perp}\right)\right\|^{2}+2 \sum_{\alpha=1}^{k}\left\|h\left(\mathcal{D}^{\theta}, \mathcal{D}_{\alpha}^{T}\right)\right\|^{2} \\
& +2 \sum_{a=1}^{l}\left\|h\left(\mathcal{D}^{\theta}, \mathcal{D}_{a}^{\perp}\right)\right\|^{2}+2 \sum_{1 \leq \alpha<\beta \leq k}\left\|h\left(\mathcal{D}_{\alpha}^{T}, \mathcal{D}_{\beta}^{T}\right)\right\|^{2} \\
& +2 \sum_{1 \leq a<b \leq l}\left\|h\left(\mathcal{D}_{a}^{\perp}, \mathcal{D}_{b}^{\perp}\right)\right\|^{2}+2 \sum_{\alpha=1}^{k} \sum_{a=1}^{l}\left\|h\left(\mathcal{D}_{\alpha}^{T},, \mathcal{D}_{a}^{\perp}\right)\right\|^{2} .
\end{aligned}
$$

Here by Corollary 5.5 and the fact that $\left(\mathcal{D}^{\theta}, \mathcal{D}_{a}^{\perp}\right)$-mixed geodesic, we have $h\left(\mathcal{D}^{\theta}, \mathcal{D}_{\alpha}^{T}\right)=0, h\left(\mathcal{D}^{\theta}, \mathcal{D}_{a}^{\perp}\right)=0$, $h\left(\mathcal{D}_{\alpha}^{T}, \mathcal{D}_{a}^{\perp}\right)=0$ and $h\left(\mathcal{D}_{\alpha}^{T}, \mathcal{D}_{\beta}^{T}\right)=0$ for $1 \leq \alpha<\beta \leq k$ and $1 \leq a \leq l$. So we obtain

$$
\begin{aligned}
\|h\|^{2} \geq & \left\|h\left(\mathcal{D}^{\theta}, \mathcal{D}^{\theta}\right)\right\|^{2}+\sum_{\alpha=1}^{k}\left\|h\left(\mathcal{D}_{\alpha}^{T}, \mathcal{D}_{\alpha}^{T}\right)\right\|^{2} \\
& +\sum_{a=1}^{l}\left\|h\left(\mathcal{D}_{a}^{\perp}, \mathcal{D}_{a}^{\perp}\right)\right\|^{2}
\end{aligned}
$$

In view of (3.6), we have

$$
\begin{aligned}
\|h\|^{2} \geq & \sum_{\alpha=1}^{k} g\left(h\left(\mathcal{D}_{\alpha}^{T}, \mathcal{D}_{\alpha}^{T}\right), N \mathcal{D}^{\theta}\right)^{2}+\sum_{\alpha=1}^{k} \sum_{b=1}^{l} g\left(h\left(\mathcal{D}_{\alpha}^{T}, \mathcal{D}_{\alpha}^{T}\right), F \mathcal{D}_{b}^{\perp}\right)^{2} \\
& +\sum_{a=1}^{l} g\left(h\left(\mathcal{D}_{a}^{\perp}, \mathcal{D}_{a}^{\perp}\right), N \mathcal{D}^{\theta}\right)^{2}+\sum_{a, b=1}^{l} g\left(h\left(\mathcal{D}_{a}^{\perp}, \mathcal{D}_{a}^{\perp}\right), F \mathcal{D}_{b}^{\perp}\right)^{2}
\end{aligned}
$$

Hence by (5.30), we know

$$
\sum_{\alpha=1}^{k} \sum_{b=1}^{l} g\left(h\left(\mathcal{D}_{\alpha}^{T}, \mathcal{D}_{\alpha}^{T}\right), F \mathcal{D}_{b}^{\perp}\right)^{2}=0
$$

Thereby, we arrive

$$
\|h\|^{2} \geq \sum_{\alpha=1}^{k} g\left(h\left(\mathcal{D}_{\alpha}^{T}, \mathcal{D}_{\alpha}^{T}\right), N \mathcal{D}^{\theta}\right)^{2}+\sum_{a=1}^{l} g\left(h\left(\mathcal{D}_{a}^{\perp}, \mathcal{D}_{a}^{\perp}\right), N \mathcal{D}^{\theta}\right)^{2}
$$


In view of decomposition (3.5) and (3.6) the inequality (6.5) can be explicitly written as follows

$$
\begin{aligned}
\|h\|^{2} \geq & \sum_{\alpha=1}^{k}\left\{\sum_{i, j=1}^{m_{\alpha}} \sum_{t=1}^{d} g\left(h\left(e_{i}^{\alpha}, e_{j}^{\alpha}\right), \csc \theta N e_{t}^{*}\right)^{2}\right\} \\
& +\sum_{a=1}^{l}\left\{\sum_{r, s=1}^{n_{a}} \sum_{t=1}^{d} g\left(h\left(w_{r}^{a}, w_{s}^{a}\right), \csc \theta N e_{t}^{*}\right)^{2}\right\} .
\end{aligned}
$$

By (5.31) and the fact that $M$ is $\left(\mathcal{D}^{\theta}, \mathcal{D}_{a}^{\perp}\right)$-mixed geodesic for $1 \leq a \leq l$, we have

$$
\sum_{r, s=1}^{n_{a}} \sum_{t=1}^{d} g\left(h\left(w_{r}^{a}, w_{s}^{a}\right), \csc \theta N e_{t}^{*}\right)^{2}=\csc ^{2} \theta \sum_{r, s=1}^{n_{a}} \sum_{t=1}^{d} g\left(T e_{t}^{*}\left(\ln \sigma_{a}\right) g\left(w_{r}^{a}, w_{s}^{a}\right)\right)^{2} .
$$

By Remark 6.1 and (3.3), we obtain

$$
\sum_{r, s=1}^{n_{a}} \sum_{t=1}^{d} g\left(h\left(w_{r}^{a}, w_{s}^{a}\right), \csc \theta N e_{t}^{*}\right)^{2}=\cot ^{2} \theta \sum_{r, s=1}^{n_{a}} \sum_{t=1}^{d}\left(e_{t}^{*}\left(\ln \sigma_{a}\right) g\left(w_{r}^{a}, w_{s}^{a}\right)\right)^{2} .
$$

By direct calculation, we get

$$
\sum_{r, s=1}^{n_{a}} \sum_{t=1}^{d} g\left(h\left(w_{r}^{a}, w_{s}^{a}\right), \csc \theta N e_{t}^{*}\right)^{2}=\cot ^{2} \theta\left\|\nabla\left(\ln \sigma_{a}\right)\right\|^{2} n_{a} .
$$

On the other hand, by (5.25) and Remark 6.1, we have

$$
\begin{aligned}
& \sum_{i, j=1}^{m_{\alpha}} \sum_{t=1}^{d} g\left(h\left(e_{i}^{\alpha}, e_{j}^{\alpha}\right), \csc \theta N e_{t}^{*}\right)^{2} \\
& =\csc ^{2} \theta \sum_{i, j=1}^{m_{\alpha}} \sum_{t=1}^{d}\left(-e_{t}^{*}\left(\ln f_{\alpha}\right) g\left(e_{i}^{\alpha}, F e_{j}^{\alpha}\right)+T e_{t}^{*}\left(\ln f_{\alpha}\right) g\left(e_{i}^{\alpha}, e_{j}^{\alpha}\right)\right)^{2} \\
& =\csc ^{2} \theta \sum_{t=1}^{d}\left\{\sum_{i, j=1}^{z_{\alpha}}\left(-e_{t}^{*}\left(\ln f_{\alpha}\right) g\left(e_{i}^{\alpha}, F e_{j}^{\alpha}\right)+\cos \theta e_{t}^{*}\left(\ln f_{\alpha}\right) g\left(e_{i}^{\alpha}, e_{j}^{\alpha}\right)\right)^{2}\right. \\
& \left.+\sum_{i, j=z_{\alpha}+1}^{m_{\alpha}}\left(-e_{t}^{*}\left(\ln f_{\alpha}\right) g\left(e_{i}^{\alpha}, F e_{j}^{\alpha}\right)+\cos \theta e_{t}^{*}\left(\ln f_{\alpha}\right) g\left(e_{i}^{\alpha}, e_{j}^{\alpha}\right)\right)^{2}\right\} \\
& =\csc ^{2} \theta \sum_{t=1}^{d}\left(e_{t}^{*}\left(\ln f_{\alpha}\right)\right)^{2}\left\{\sum_{i, j=1}^{z_{\alpha}}\left((\cos \theta-1) g\left(e_{i}^{\alpha}, e_{j}^{\alpha}\right)\right)^{2}\right. \\
& \left.+\sum_{i, j=z_{\alpha}+1}^{m_{\alpha}}\left((\cos \theta+1) g\left(e_{i}^{\alpha}, e_{j}^{\alpha}\right)\right)^{2}\right\} .
\end{aligned}
$$

Upon direct calculation, we find

$$
\begin{aligned}
& \sum_{i, j=1}^{m_{\alpha}} \sum_{t=1}^{d} g\left(h\left(e_{i}^{\alpha}, e_{j}^{\alpha}\right), \csc \theta N e_{t}^{*}\right)^{2} \\
& =\csc ^{2} \theta\left\|\nabla\left(\ln f_{\alpha}\right)\right\|^{2}\left((1+\cos \theta)^{2} m_{\alpha}-4 \cos \theta z_{\alpha}\right) .
\end{aligned}
$$

If we use (6.7) and (6.8) in the inequality (6.6), we find the inequality (6.1). Now, in view of Lemmas 5.1 and 5.2 , the inequality sign in (6.1) holds identically if and only if

$$
h\left(\mathcal{D}^{\theta}, \mathcal{D}^{\theta}\right)=\{0\}, \quad g\left(h\left(\mathcal{D}_{a}^{\perp}, \mathcal{D}_{a}^{\perp}\right), F \mathcal{D}_{b}^{\perp}\right)=\{0\} .
$$

Since $M^{\theta}$ is totally geodesic in $M$, it follows from the first condition in (6.9) that $M^{\theta}$ is totally geodesic in $\bar{M}$. 


\section{Acknowledgments}

All authors except the third one are supported by 1002-Scientific and Technological Research Projects Funding Program of TUBITAK project number 119F207. Moreover, the second author is supported by the Scientific Research Projection Coordination Unit of İstanbul University with project numbered 25839.

\section{References}

[1] Adati, T. : Submanifolds of an almost product Riemanian manifold. Kodai Math J. 4, 327-343 (1981).

[2] Al-Solamy, F.R., Khan, M.A.: Warped product submanifolds of Riemannian product manifolds. Hindawi Publishing Corporation Abstract and Applied Analysis. Article ID 724898, 12 pages (2012).

[3] Atçeken, M.: Warped product semi-slant submanifolds in locally Riemannian product manifolds. Bull. Austral. Math. Soc. 77 (2), 177-186 (2008).

[4] Atçeken, M.: Warped Product semi-invariant submanifolds in locally decomposable Riemannian Manifolds. Hacet. J. Math. Stat. 40 (3), 401-407 (2011).

[5] Atçeken, M.: Geometry of warped product semi-invariant submanifolds of a locally Riemannian product manifolds. Serdica Math. J. 35, 273-289 (2009).

[6] Bejan, C.L.: Almost semi-invariant submanifolds of locally product Riemannian manifolds. Bull. Math. de la Soc. Sci. Math. de la R. S. de Roumanie Tome. 32 (80), No. 1, 3-9 (1988).

[7] Bejancu, A.: Semi-invariant submanifolds of locally product Riemannian manifolds. An. Univ. Timişoara Ser. Ştiint. Math. Al. 22(1-2), 3-11 (1984).

[8] Bishop, R. L., O’Neill, B.: Manifolds of negative curvature. Trans. Amer. Math. Soc. 145(1), 1-49 (1969).

[9] Chen, B. Y.: Geometry of warped product submanifolds in Kaehler manifolds. Monatsh Math. 133, 177-195 (2001).

[10] Chen, B. Y., Dillen, F.: Optimal Inequalities For Multiply Warped Product Submanifolds. Int. Electron. J. Geom. 1(1), 1-11 (2008).

[11] Chen, B.Y.: Differential geometry of warped product manifolds and submanifolds. World Scientific. (2017).

[12] Dillen, F., Nölker, S.: Semi-paralellity multi rotation surfaces and the helix property. J. Reine. Angew. Math. 435, 33-63 (1993).

[13] Gerdan Aydın, S., Taștan, H. M., Traore, M., Ülker, Y.: Biwarped product submanifolds with a slant base factor. (Preprint).

[14] Liu, X., Shao, F. M.: Skew semi-invariant submanifolds of locally product manifold. Portugalie Math. 56, 319-327 (1999).

[15] Li, H., Liu, X.: Semi-slant submanifolds of a locally product manifold. Georgian Math. J. 12, 273-282 (2005).

[16] O'Neill, B.: Semi-Riemannian geometry with applications to relativity. Academic Press. San Diego (1983).

[17] Şahin, B.: Slant submanifolds of an almost product Riemannian manifold. J. Korean Math. Soc. 43, 717-732 (2006).

[18] Şahin, B.: Warped Product semi-slant submanifolds of a locally product Riemannian manifold. Studia Sci. Math. Hungar. 46(2), 169-184 (2009).

[19] Şahin, B.: Warped product semi-invariant submanifolds of a locally product Riemannian manifold. Bull. Math. Soc. Sci. Math. Roumanie. 49(97), 4, 383-394 (2006).

[20] Taştan, H. M.: Warped product skew semi-invariant submanifolds of order 1 of a locally product Riemannian manifold. Turk. J. Math. 39, 453-466 (2015).

[21] Taștan, H. M., Özdemir, F.: The geometry of hemi-slant submanifolds of a locally product Riemannian manifold. Turk. J. Math. 39, 268-284 (2015).

[22] Uddin, S., Mihai, A., Mihai, I., Al-Jedani, A.: Geometry of bi-warped product submanifolds of locally product Riemannian manifolds. RACSAM. 114(42), (2020). https://doi.org/10.1007/s13398-019-00766-6.

[23] Ünal, B.: Multiply warped products. J. Geom. Phys. 34(3), 287-301 (2000).

[24] Xu, S., Ni, Y.: Submanifolds of product Riemannian manifolds. Acta Mathematica Scientia. 20B(2), 213-218 (2000).

[25] Yano, K., Kon, M.: Structures on manifolds. World Scientific, Singapore (1984).

\section{Affiliations}

\section{MOCTAR TRAORE}

ADDRESS: İstanbul University, Dept. of Mathematics, 34134, İstanbul-Turkey.

E-MAIL: tramoct@gmail.com

ORCID ID: 0000-0003-2132-789X

\section{HAKAN METE TAŞTAN}

ADDRESS: İstanbul University, Dept. of Mathematics, 34134, İstanbul-Turkey.

E-MAIL: hakmete@istanbul.edu.tr

ORCID ID: 0000-0002-0773-9305

\section{SiBEL GERDAN AYDIN}

ADDRESS: İstanbul University, Dept. of Mathematics, 34134, İstanbul-Turkey.

E-MAIL: sibel.gerdan@istanbul.edu.tr

ORCID ID: 0000-0001-5278-6066 